$\mathrm{DOE} / \mathrm{ER} / 40561--33$

DOE/ER/40561

DE92 004389

\title{
Chiral Symmetry, Axial Anomaly \\ and the Structure of Hot QCD
}

\author{
Tetsuo Hatsuda \\ Institute for Nuclear Theory, University of Washington \\ $H N-12$, Seattle, Washington 98195, USA
}

\section{PREPARED FOR THE U.S. DEPARTMENT OF ENERGY UNDER GRANT DE-FG06-90ER40561}

This report was prepared as an account of work sponsored by the United States Government. Neither the United States nor any agency thereof, nor any of their employees, makes any warranty, express or implied, or assumes any legal liability or responsibility for the accuracy, completeness, or usefulness of any information, apparatus, product, or process disclosed, or represents that its use would not infringe privately owned rights. Reference herein to any specific commercial product, process, or service by trade name, mark, manufacturer, or otherwise, does not necessarily constitute or imply its endorsement, recommendation, or favoring by the United States Government or any agency thereof. The views and opinions of authors expressed herein do not necessarily siate or reflect those of the United States Government or any agency thereof. 


\title{
CHIRAL SYMMETRY, AXIAL ANOMALY AND THE STRUCTURE OF HADRONS
}

\author{
Tetsuo HATSUDA \\ Institute for Nuclear Theory, University of Washington \\ $H N-12$, Seattle, Washington 98105, USA
}

\begin{abstract}
This lecture is composed of three parts. [I] Heavy quark and gluon contents of light hadrons, [II] anomalous gluon content of the nucleon, and [III] hot and dense QCD. Non-valence structures of nucleon due to the OZI violation are extensively discussed in [I] and [II], and non-perturbative aspects of the quark-gluon plasma are reviewed in [III].
\end{abstract}

Invited Lectures given at "Winter Meeting on Intermediate Energy Nuclear Physics" (Mt. Soirak, Korea, Feb. 26 - March 1, 1991). 


\section{[1] Heavy Quark and Gluon Contents of Light Hadrons}

\section{Introduction}

Effects of the flavor mixing have been one of the main subjects of hadron physics. It is known that the mixing is small in the meson sector, which is summarized as the celebrated OZI-rule[1]; a few exceptions include the system of $\eta$ and $\eta^{\prime}$ mesons where the axial anomaly gives a large violation of the OZI-rule. Recently there has arisen a growing interest in the OZI-violating processes in the baryon sector. The relevant observables include the $\pi-N$ and $K-N$ sigma terms $\left(\Sigma_{\pi N}, \Sigma_{K N}\right)[2]$, the quark-spin content of the proton i.e. the flavor singlet axial-charge $g_{A}^{0}$ of the nucleon[3], intrinsic charm quarks in the nucleon suggested by the $J / \psi$ - and open-charm production in hadron-nucleus collisions [4], and the strange-quark contribution to the Higgs-nucleon coupling $[17,18]$. Other processes in hadron reactions which favor or disfavor the OZI violation in the nucleon are also suggested.[5]

The purpose of this chapter is to give a realistic estimate of the OZI-violation in the baryon sector on the basis of an effective Lagrangian successful to describe the OZI-violation in the meson sector.

Before entering the detailed discussion, let us breifly summarize the reason why the observables mentioned above are interesting. The $\pi-N$ sigma term $\Sigma_{\pi N}$, which is proportional to the isospin-even $\pi-N$ scattering amplitude with vanishing external four momenta, is given by the nucleon matrix element of the scalar operator

$$
\Sigma_{\pi N}=\hat{m}\langle\bar{u} u+\bar{d} d\rangle_{N} .
$$

Here $\hat{m} \equiv\left(m_{u}+m_{d}\right) / 2$ is the average value of the current masses of $u$ and $d$ quarks. (We use the non-covariant normalization for the state vector $\left\langle p^{\prime} \mid p\right\rangle=(2 \pi)^{3} \delta^{3}\left(\mathbf{p}^{\prime}-\mathbf{p}\right)$ throughout this paper unless otherwise is stated.) Although the "empirical" value of $\Sigma_{\pi N}$ is still uncertain, the latest analysis shows $\Sigma_{\pi N}=45 \mathrm{MeV}$ with an estimated error $\pm 12 \mathrm{MeV}$.[8] Now, if one assumes the validity of the 1st order expansion by the current quark masses $\left(m_{u, d, s}\right)$, one can relate $\Sigma_{\pi N}$ to the OZI violating strangeness content of nucleon (see e.g. [2]),

$$
\Sigma_{\pi N}=\frac{1}{1-y} \frac{1}{m_{s} / \hat{m}-1}\left(M_{\equiv}+M_{\Sigma}-2 M_{N}\right)
$$

with

$$
y=\frac{2\langle\bar{s} s\rangle_{N}}{\langle\bar{u} u+\bar{d} d\rangle_{N}} .
$$

Intuitively, $y$ represents the probability to find $s$ or $\bar{s}$ in nucleon. One sees that $y$ must be as large as $0.42_{-0.21}^{+0.12}$ to reproduce the empirical value $\Sigma_{\pi N}=(45 \pm 12) \mathrm{MeV}$. 
1 This suggests that the violation of the OZI-rule could be much larger in baryons than that in mesons, while there are other possibilities where eq.(1.2) is substantially modified by the inclusion of the higher order analytic and non-analytic terms of $m_{s}{ }^{2}$

The EMC data [3] on the spin dependent structure function of the proton suggest that the OZI-rule is largely violated in the axial vector channel, too;

$$
\left.\left\langle\bar{s} \gamma_{\mu} \gamma_{5} s\right\rangle_{N}\right|_{\mu^{2}=10 G e V^{2}}=(-0.19 \pm 0.06) \cdot \bar{N} \gamma_{\mu} \gamma_{5} N
$$

with $\mu$ being the renormalization point of the axial current operator. $\left(\bar{N} \gamma_{\mu} \gamma_{5} N\right.$ is the normalization factor with the Dirac spinor $N$.) Although the problem is still controversial (see e.g. [10] and the reviews [11]), it might imply that there exists a sizeable $s$-quark content with the opposite helicity to the polarization of the proton.

The mass number $(A)$ dependence $A^{\alpha\left(x_{F}\right)}$ of the $J / \psi$ and $\psi^{\prime}$ production from nuclei is known to have two components, which are characterized by the Feynman $x_{F}$ : For small $x_{F}, \alpha\left(x_{F}\right) \simeq 1$, i.e., the cross section behaves like $A^{1}$ which is consistent with the picture of the production through a hard process; the other component behaves like $A^{2 / 3}$, which dominates $A^{1}$-component for large $x_{F}$. [12] The latter may be accounted for in terms of the so-called diffractive dissociation in which the intrinsic charm of the nucleon plays an essential role.[4] This intrinsic charm, which can be only described non-perturbatively, should be attributed to an OZI-violation in the nucleon wavefunction and has several other implications.[13]

The Higgs-nucleon coupling constant also depends on the sea-quarks in the nucleon. As was first addressed by Shifman, Vainstein and Zakharov (SVZ) [14], the coupling is mostly determined by the heavy sea-quarks $(c, b, t)$. It was, however, subsequently questioned by T. P. Cheng[17] and H. Y. Cheng[18] who pointed out that the coupling constant would be enhanced by a factor 2 to 3 if the strangeness content of the nucleon is as large as suggested in the first order chiral perturbation, i.e., $y \sim 0.5$.

The above examples tell us the importance of a quantitative estimation of the heavy as well as the light quark contents of baryons. This is possible for the quark content in the scalar channel $\langle\bar{q} q\rangle_{B}$, since one can use a sum rule such as the QCD trace anomaly [15] for the heavy quarks and the Feynman-Hellman (FH) theorem [16] for the light quarks.

The light-quark content of baryons obtained by the $\mathrm{FH}$ theorem is given in $[6,7]$ and will be briefly summarized in sections 2 and 3 ;

$$
\left\langle\bar{q}_{i} q_{i}\right\rangle_{B}=\frac{\partial M_{B}}{\partial m_{i}}
$$

\footnotetext{
${ }^{1}$ It is also noteworthy that such a large strangeness content implies that the nucleon mass in the chiral limit is as small as $(660 \mp 161) \mathrm{MeV}$, provided that the first order expansion with respect to the current masses is valid.

${ }^{2}$ For example, the inclusion of the leading non-analytic terms in the chiral perturbation gives an enhancement of order $10 \mathrm{MeV} ; \Sigma_{x N}=(35 \pm 5) /(1-y) \mathrm{MeV}[9]$. This enhancement tends to make $y$ smaller than the lowest order result. See section 3 for the comparison of this result with that in our effective Lagrangian approach.
} 
where $i=(u, d, s), M_{B}$ is the baryon mass and $m_{i}$ is the current mass of the $i$-th quark. ${ }^{3}$ To get $M_{B}$ in terms of $m_{i}$, we take a chiral quark model where the constituent quark masses $[19,20]$ are generated by the spontaneous breaking of chiral symmetry $\left(\mathrm{SB}_{\chi} \mathrm{S}\right)$. The generalized $S U_{f}(3)$-Nambu-Jona-Lasinio ( $\mathrm{NJL}$ ) model [21] incorporating the axial anomaly $[22,23]$ will be adopted to describe $\mathrm{SB} \chi \mathrm{S}$ : The term responsible for the flavor mixing is the 't Hooft vertex induced by the axial anomaly $[24,25,26,27]$.

$\left\langle\bar{q}_{i} q_{i}\right\rangle_{B}$ calculated with (1.5) shows small strangeness content $(y \simeq 0.12)$ and a large $\Sigma_{\pi N}(\simeq 50 \mathrm{MeV})$ are compatible [6], which has been suggested by several authors on the basis of several models $[24,25,26,28]$. The non-linear $m$, dependence of the physical quantities $[24,26,29]$ and the short-range interaction due to the one-gluon exchange [6] are essential for this fact. ${ }^{4}$ Then we will go ahead and calculate the anomalous quark contents or the sea-quark contents of the other members of the octet and decuplet. In general, the anomalous quark contents due to the axial anomaly is small but non-negligible.

The heavy quark contents $(c, b, t)$ of static baryons such as $\langle\bar{c} c\rangle_{N}$ can be extracted from the trace anomaly in QCD;

$$
M_{B}=\left\langle\Theta_{\mu}^{\mu}\right\rangle_{B}=\sum_{q} m_{q}\left(1+\gamma_{m}\right)\langle\bar{q} q\rangle_{B}+\frac{\beta}{4 \alpha_{s}}\left\langle G^{2}\right\rangle_{B}
$$

where $q$ runs for the heavy quarks $(q=h=c, b, t)$ as well as light quarks $(q=l=u, d, s)$. $\beta=-\left(11-2 N_{f} / 3\right) \alpha_{s}^{2} /(2 \pi)+\cdots$ is the Callan-Symanzik $\beta$-function and $\gamma_{m}$ is the mass anomalous dimension. ${ }^{5}$

The heavy-quark contents will be evaluated by the combined use of the FH theorem eq.(1.5) and the heavy quark mass expansion [31] in section 4 . The resulting intrinsic charm content of the nucleon reads

$$
\frac{\langle\bar{c} c\rangle_{N}}{\langle\bar{u} u+\bar{d} d\rangle_{N}} \simeq 5 \times 10^{-3}
$$

which may be compared with the value $\left(3 \times 10^{-3}\right)$ deduced from the analysis cf the charm structure function of the nucleon.[32] We also show that the heavy quark contenis are larger in the decuplet baryons than in the octet.

As a straightforward application of the calculation of $\langle\bar{q} q\rangle_{B}$, we will evaluate the Higgs-baryon coupling constant $g_{\phi B B}$ in section 5 . It is shown that $g_{\phi B B}$ depends crucially on the value of $\langle\bar{s} s\rangle_{B}$ and our result

$$
g_{\phi N N} \simeq 1.2 \times 10^{-3}=0.32 \cdot\left(\sqrt{2} G_{F}\right) \cdot M_{N},
$$

\footnotetext{
${ }^{3}$ Note that if we use the covariant normalization for the state vector, the r.h.s. of (1.5) reads $\partial M_{B}^{2} / \partial m_{i}$.

${ }^{4}$ The effect of the one-gluon exchange on $\Sigma_{\pi N}$ was first considered by Nelson [30] using a naive constituent-quark model.

${ }^{5}$ At the energy scale of order $1 \mathrm{GeV}, \gamma_{m}$ takes 0.22 if one uses the one-loop result $\gamma_{m}=2 \alpha_{\mathrm{s}} / \pi$ with $\alpha_{s}(1 \mathrm{GeV}) \simeq 0.35$. The effect of it can, however, be absorbed into a modification of the masses of the current quarks $m_{q} \rightarrow m_{q}^{*} \equiv m_{q}\left(1+\gamma_{m}\right)$, so one may just regard it as $O(20 \%)$ uncertainty of the current quark masses.
} 
lies in the middle of the previous two extreme estimates using $y=0[14]$ and $y \simeq 0.5$ $[17,18]$. (Here $G_{F}$ is the Fermi constant.)

Finally, we will briefly discuss the quark contents of the pion and the kaon using the NJL model in section 6 . The heavy quark content and hence the gluon content in the pion vanish in the chiral limit $\left(m_{u, d} \rightarrow 0\right)$ as is expected from the NambuGoldstone realization of the chiral symmetry. The realistic values off the chiral limit are found to be

$$
\frac{\left|\langle\bar{s} s\rangle_{\pi}\right|}{\langle\bar{u} u+\bar{d} d\rangle_{\pi}}<4.5 \times 10^{-4} \text { and } \frac{\langle\bar{c} c\rangle_{\pi}}{\langle\bar{u} u+\bar{d} d\rangle_{\pi}} \simeq 3.0 \times 10^{-4}
$$

which are much smaller than the corresponding quantities for baryons. This difference is nothing but a consequence of the Nambu-Goldstone nature of pion. In the final section, we will give a brief summary and concluding remarks.

\section{The dynamical mass and the constituent quark model}

The constituent quark model (CQM) $[19,20]$ has a great success in reproducing the mass spectrum of baryons. Therefore, it should be a good starting point for the study of the quark content of baryons. An apparent difficulty in applying CQM to the problem of the quark-content is that the relation of the constituent quark masses $M_{u, d, s}$ with the current quark masses $m_{u, d, s}$ is unknown: $M_{u, d, s}$ are usually the fitting parameters in the model. This difficulty is overcome by taking the picture advocated by several authors that $M_{u, d, s}$ are identified with the dynamical masses generated by the spontaneous breaking of chiral symmetry. [33, 34, 35, 36] Although one does not know the precise mechanism of the chiral symmetry breaking in QCD yet, it can be well described by the effective Lagrangian of the Nambu-Jona-Lasinio (NJL) type. $[35,36]^{6}$

Following Goldman and Haymaker [33], we decompose the low energy effective Lagrangian into three parts;

$$
\mathcal{L}=\mathcal{L}_{N J L}+\mathcal{L}_{O G E}+\mathcal{L}_{C O N F}
$$

Here $\mathcal{L}_{N J L}$ is the NJL model to be specified later and is understood to be responsible for chiral symmetry breaking and the flavor mixing. $\mathcal{L}_{O G E}$ and $\mathcal{L}_{C O N F}$ denote the short-range interaction due to the one-gluon-exchange (OGE) and the force responsible for confinement, respectively. Our basic assumption is that the dynamics may be treated in two steps as suggested by Goldman and Haymaker: First $\mathcal{L}_{N J L}$ is turned on, hence the dynamical quark masses are generated, which are identified with the constituent quark masses. Then the short-range and long-range interactions

\footnotetext{
${ }^{6} \mathrm{~A}$ full review of the NJL model may be found in [37].
} 
represented by $\mathcal{L}_{O G E}$ and $\mathcal{L}_{C O N F}$ are put on as relatively small perturbation between these constituent quarks.

Now let us write down the explicit form of $\mathcal{L}_{N J L}[24,25]$;

$$
\begin{aligned}
\mathcal{L}_{N J L}= & \bar{q}(i \gamma \cdot \partial-\mathbf{m}) q+\sum_{a=0}^{8} \frac{g_{s}}{2}\left[\left(\bar{q} \lambda_{a} q\right)^{2}+\left(\bar{q} i \lambda_{a} \gamma_{5} q\right)^{2}\right] \\
& +g_{D}\left[\operatorname{det} \bar{q}_{i}\left(1-\gamma_{5}\right) q_{j}+h . c .\right]
\end{aligned}
$$

where the quark field $q_{i}$ has three colors $\left(N_{c}=3\right)$ and three flavors $\left(N_{f}=3\right)$, and $\lambda_{a}$ $(a=0 \sim 8)$ are the Gell-Mann matrices with $\lambda_{0}=\sqrt{\frac{2}{3}} 1$. The second term is the explicit $S U_{V}(3)\left(S U_{f}(3)\right)$-breaking part with $\mathrm{m}=\operatorname{diag}\left(m_{u}, m_{d}, m_{s}\right)$ being the current quark mass matrix.

As can be easily seen, the second term does not mix the flavors [38]; in other words, it represents an effective Lagrangian in the large $N_{c}$ limit of QCD where all the virtual quark-loops can be neglected. Since the origin of such an effective 4fermion interaction has been discussed by many authors, we do not repeat it here and just quote some references $[35,36]$. The third term ('t Hooft term) represents the major non-leading term of the large $N_{c}$ expansion i.e. the axial anomaly: This term has the $S U_{L}(3) \otimes S U_{R}(3)$ invariance but breaks the $U_{A}(1)$ symmetry and is responsible for the mixing of different flavors (OZI violation). For example, it induces effective 4fermi vertices such as $(\bar{u} u)(\bar{s} s)\langle\bar{d} d\rangle_{0}$ and $-\left(\bar{u} i \gamma_{5} u\right)\left(\bar{s} i \gamma_{5} s\right)\langle\bar{d} d\rangle_{0}\left(\langle\cdot\rangle_{0}\right.$ being the vacuum expectation value); the former contributes to the flavor mixing in the scalar mesons, while the latter contributes to the mixing in the pseudoscalar mesons. One can also regard this determinant-term as an effective interaction induced by instanton [39]. The same kind of effective Lagrangian was first introduced by Kobayashi and Maskawa [22] as a clue to solve the $\eta^{\prime}$-mass problem and has been examined in the long-wavelength limit by Schechter et al [23]. ${ }^{7}$

Lagrangian (2.2) has several parameters; the current quark masses $\mathrm{m}=\operatorname{diag}\left(m_{u}, m_{d}, m_{s}\right)$, the coupling constants $g_{s}$ and $g_{D}$, and the momentum cutoff $\Lambda$ characterizing the scale of the chiral symmetry breaking. (For $m_{u}$ and $m_{d}$, we assume the $S U_{f}(2)$-invariance and take $\hat{m}=\left(m_{u}+m_{d}\right) / 2$ as a parameter.) Our strategy is to fix these parameters in the meson sector and apply the same Lagrangian and the parameters to the baryon sector.

The parameters were determined to reproduce the four basic quantities $m_{\pi}=138$ $\mathrm{MeV}, f_{\pi}=93 \mathrm{MeV}, m_{K}=495.7 \mathrm{MeV}$ and $n_{\eta^{\prime}}=957.5 \mathrm{MeV}^{\prime} .\left(g_{S}, \Lambda\right), m_{s}$ and $g_{D}$ are fixed essentially by $\left(m_{\pi}, f_{\pi}\right), m_{k}$ and $m_{\eta^{\prime}}$ respectively. The allowed upper limit of $\hat{m}$ which can reproduce $f_{\pi}$ and $m_{\pi}$ simultaneously turns out to be $5.8 \mathrm{MeV}$ in our model. By combining this value with the known lower limit for $\hat{m}(1 \mathrm{GeV})$ by other means, we adopt $5.5 \mathrm{MeV}$ as a standard value for $\hat{m}$ at $1 \mathrm{GeV}$ scale here. ${ }^{8}$ The resulting

\footnotetext{
${ }^{7} \mathrm{An} S U_{f}(2)$-version of the 't Hooft vertex was extensively examined in [36] without recourse to the long-wave length limit.

${ }^{8} \hat{m}$ at $1 \mathrm{GeV}$ scale is determined by several means (the meson and baryon spectra, the low energy
} 
parameter set reads ${ }^{9}$

$$
\Lambda=631.4 \mathrm{MeV}, \quad g_{S} \Lambda^{2}=3.67, \quad g_{D} \Lambda^{5}=-9.29, \quad m_{s}=135.7 \mathrm{MeV} .
$$

Here we have used a three-momentum cutoff scheme. If one uses the Lorentz-invariant cutoff, it has a value $\simeq 1 \mathrm{GeV}$ (see e.g. the detailed study [41]) which is identified with the typical scale of the chiral symmetry breaking and also the optimum scale $\mu$ mentioned before.

The basic quantity for our purpose is the dynamical quark masses which are obtained by solving the coupled gap equation[25];

$$
M_{u}=m_{u}-2 g_{0}\langle\bar{u} u\rangle_{0}-2 g_{D}\langle\bar{d} d\rangle_{0} \cdot\langle\bar{s} s\rangle_{0},
$$

and the equations for $M_{d}$ and $M_{s}$ obtained by the cyclic permutation of $u, d$ and $s$, where $\left\langle\bar{q}_{i} q_{i}\right\rangle_{0}(\mathrm{i}=\mathrm{u}, \mathrm{d}, \mathrm{s})$ is the $\mathrm{i}$-th quark condensate being a function of $M_{i}$. The 3rd term of the gap equation eq. (2.4) originates from the 't Hooft term, which is about $10 \%$ of the total constituent mass. The solution of the gap equation reads $M_{u}=M_{d}=335$ $\mathrm{MeV}$ and $M_{s}=527 \mathrm{MeV}$, which are quite consistent with the phenomenological masses used in CQM [20]. The above coupled equation relates $M_{i}$ with $m_{i}$ in a non-linear way. As for $m_{u, d}$, the linear approximation of $M_{i}$ is legitimate, but the legitimacy does not hold for $m_{s}$. In fact, it can be proved that there is a definite converge dius $(\sim 50 \mathrm{MeV})$ for tise expansion by $m_{i}$ in this model, which implies that one cannot expand the physical quantities by $m_{s}(\sim 150 \mathrm{M \epsilon} \cdot \mathrm{V})[42,7]$.

In Table 1, we have listed the several physical quantities predicted by the model in the meson sector and their empirical values.[7] The agreement is good in the $10-15 \%$ level.

A few comments are in order here.

(i) Our strategy and approximation here is not to expand the observables by the current mass especially in the strange sector since we know there is a convergence radius of such expansion as we mentioned above.

(ii) The meson-loop contributions, which we have neglected here, are expected to be relatively small. This can be explicitly shown by calculating the quark selfenergy due to the meson loops which gives at most $10 \%$ correction. Furthermore, this is consistent with an observation by Chan and Haymaker [43]: If one starts with the $S U_{V}(3)$ asymmetric vacuum $\left(\langle\bar{u} u\rangle_{0} \neq\langle\bar{s} s\rangle_{0}\right)$ and takes into account both the non-analytic and analytic terms due to the meson loops, the loop-contribution is rather small. On the other hand, if one starts with the $S U_{V}(3)$ symmetric vacuum $\left(\langle\bar{u} u\rangle_{0}=\langle\bar{s} s\rangle_{u}\right)$ and takes into account only the leading non-analytic terms, the

current matrix elements, QCD sum rules, et.c) which are summarized in Appendix A of ref.[40] (See also [9]). The values determined from the evaluation of the current matrix elements at low energies are centered around $5.5 \mathrm{MeV}$ while the results using the QCD sum rules are around $7 \mathrm{MeV}$. All the results are, however, consistent with the range $5-9 \mathrm{MeV}$ for $\hat{m}$.

${ }^{9} \mathrm{~W}$ e have made a finer tuning of the parameters than those made in $[6,7,25]$, hence the parameters here are slig'stly different from those quoted there. 
contribution is sizable as is well known in the chiral perturbation theory [45]. However, both approaches have the same tendency to improve the result obtained in the chiral limit. We will see this fact explicitly in the later sections.

(iii) In the meson sector, the confinement $\mathcal{L}_{C O N F}$ has little effect, at least, on the pion as is shown by Goldman and Haymaker [33]. The good agreement with the empirical values listed in Table 1 suggests the same situation holds even in the other mesons.

(iv) The key parameter that controls the flavor mixing is $g_{D}$, i.e., the strength of the ' $t$ Hooft interaction, which determines the OZI violation in the constituents quarks (eq.(2.4)) and also in the $\bar{q} q$ systems. A dimensionless measure of its strength is the ratio $r \equiv g_{D}\langle\bar{q} q\rangle_{0} / g_{s}$ which is nothing but the ratio between the effective 4fermi interaction due to the 't Hooft term and the OZI conserving vertex[25]. As we already remarked, the magnitude of $g_{D}$ is determined so as to reproduce the mass of the $\eta^{\prime}$-meson and the resultant $\eta-\eta^{\prime}$ mixing angle agrees with the empirical value $\sim-20^{\circ}$. In fact, both the mass and the mixing angle in the pseudoscalar channel are sensitive to $g_{D}$, since OZI violation works most effectively in the pseudo-scalar channel. The small mixing angle implies that $\eta$ and $\eta^{\prime}$ are an almost $S U(3)$-octet ard singlet state, i.e. $(\bar{u} u+\bar{d} d-2 \bar{s} s) / \sqrt{6}$ and $(\bar{u} u+\bar{d} d+\bar{s} s) / \sqrt{3}$, respectively. On the other hand, because of the sign difference of the 't Hooft vertex in the scalar channel [25] from that in the pseudo-scalar channel, the scalar mesons are close to the eigen states of the quark basis, i.e., $(\bar{u} u+\bar{d} d) / \sqrt{2}$ and $\bar{s} s .{ }^{10}$ The detailed discussion on this point is given in ref.[7] With the parameter $g_{D}$ thus determined, one obtains $r \simeq 10 \%$, which will be found to be the typical value for the rate of to the OZI violation in the baryon sector in section 3 .

The effects of confinement and OGE $\left(\mathcal{L}_{O G E}\right.$ and $\left.\mathcal{L}_{C O N F}\right)$ in the baryon mass formula are summarized in the simple mass formula in CQM (e.g. by Isgur and Karl $[20])$, in which we use our dynamical masses as constituent quark masses.[6, 7] It gives an excellent fit for the baryon masses with errors of $1 \%$, which is indispensable to extract the heavy quark contributions as presented in the later section; if not, one cannot get the reliable number for them. ${ }^{11}$

As we have fixed all the parameters of the model, we can calculate the quark contents without free parameters.

\footnotetext{
${ }^{10}$ The mixing angle $\varphi_{\sigma}\left(\theta_{\eta}\right)$ of the scalar (pseudoscalar) mesons in Table 1 is given in the quark basis (flavor basis): Namely physical $\sigma$ and $\sigma^{\prime}$ mesons are represented in terms the non-strange sigma $\sigma_{N S} \sim(\bar{u} u+\bar{d} d) / \sqrt{2}$ and the strange sigma $\sigma_{s} \sim \bar{s} s$ as $\sigma=\sigma_{N S} \cos \varphi_{0}-\sigma_{s} \sin \varphi_{o}$ and $\sigma^{\prime}=\sigma_{N S} \sin \varphi_{\sigma}+\sigma_{s} \cos \varphi_{\sigma}$. The definition of $\theta_{\eta}$ is the same with that of the particle data group. Therefore, the small value of $\varphi_{0}\left(\theta_{\eta}\right)$ implies that the scalar (pseudoscalar) mesons are close to (far from) the ideal mixing.

${ }^{11}$ Here one should note that the instanton induced interaction in $\mathcal{L}_{N J L}$ provides the same kind of baryon mass splitting as those given by the conventional OGE [46]. Therefore we have not made a clear distinction between the twc contributions in the baryon mass formula.
} 


\section{Light quark contents of baryons}

Applying the FH theorem eq.(1.5) together with the baryon mass formula in CQM, one gets the quark contents of octet and decouplet baryons.[6, 7] The results are given in Table 2 for later use.

Typical values of the $i$-th current-quark content in the $j$-th constituent-quark denoted by $Q_{j i}$ in the paramter set (2.3) read

$$
Q_{j i} \equiv \frac{\partial M_{j}}{\partial m_{i}}=\left(\begin{array}{lll}
2.14 & 0.45 & 0.15 \\
0.45 & 2.14 & 0.15 \\
0.28 & 0.28 & 1.42
\end{array}\right) .
$$

It is easily verified that the ratio $y$ is not affected by the inclusion of $\mathcal{L}_{O G E}$ nor $\mathcal{L}_{\text {CONF }}$ and can be written only in terms of $Q_{j i}$. By putting the error coming from the uncertainty of the current mass in the range $5 \mathrm{MeV}<\hat{m}<5.8 \mathrm{MeV}$, one gets; $^{12}$

$$
y=\frac{2 Q_{u s}}{Q_{u u}+Q_{u d}}=0.12 \pm 0.01
$$

where the larger $\hat{m}$ corresponds to the smaller $y$. These numbers are as small as $r \simeq$ $10 \%$ introduced as a typical measure of the OZI violation in section 2 , whereas they are considerably smaller than $0.42_{-0.21}^{+0.12}$ expected from the 1 st order chiral perturbation without non-analytic terms (eq.(1.2)). One should remark that (i) the strength of $g_{D}$, which gives only a small $\bar{s} s$ in nucleon, is large enough to reproduce the $\eta^{\prime}$ mass and the $\eta-\eta^{\prime}$ mixing angle, and (ii) the $s$-mixing in $u\left(Q_{u s} / Q_{u u}=0.07\right)$ is much smaller than the $d$-mixing in $u\left(Q_{u d} / Q_{u u}=0.21\right)$ because of the mass suppression $M_{s}>M_{d}$.

It should be stressed that in spite of the small $y, \Sigma_{\pi N}$ has a large value ${ }^{13}$;

$$
\Sigma_{\pi N}=49 \pm 7 \mathrm{MeV},
$$

which is compared to the empirical value $\Sigma_{\pi N}=45 \mathrm{MeV}$ with an estimated error \pm 12 $\mathrm{MeV}[8]$. Since heavier quarks are difficult to excite, the proton matrix elements of $\bar{q} q$ becomes smaller as $\hat{m}$ is increased. Therefore $\Sigma_{\pi N}$ does not necessary increase proportionally to $\hat{m}$. (See also footnote 17.) We note that to give the small $y$ with large $\Sigma_{\pi N}$, the inclusion of nonlinear effects of $m_{s}[29,24,26]$ and the interaction between quarks (OGE) [6] are indispensable. Our conclusion here is qualitatively consistent with the recent investigation using the Skyrme model, which also yield a small $y$ with large $\Sigma_{\pi N}$. [28, 47]

As we mentioned in section 2, one can take a different approach from ours to evaluate $\Sigma_{\pi N}$ as is done in ref.[48]. In this case, one takes into account the leading

\footnotetext{
${ }^{12}$ At each current mass, all the other parameters are readjusted to reproduce $m_{\pi}, f_{\pi}, m_{K}$ and $m_{\eta^{\prime}}$. Thus for $\hat{m}=5.0(5.8) \mathrm{MeV}, m_{s}=128.2(138.4) \mathrm{MeV}, \Lambda=683.4(590.2) \mathrm{MeV}, g_{s} \Lambda^{2}=3.43(4.12)$ and $g_{D} \Lambda^{5}=-8.85(-9.96)$.

${ }^{13}$ Accordingly, the nucleon mass in the chiral limit needs not be so small as $660 \mathrm{MeV}$ as mentioned in footnote 3 .
} 
non-analytic terms for the current masses coming from the loops of Nambu-Goldstone bosons (but not take into account the higher order analytic terms as we are doing here). Such correction gives an enhancement of $O(10 \mathrm{MeV})$ to the 1 st order result and results in $\Sigma_{\pi N}=(40 \pm 6) \mathrm{MeV}$ for our value $y=0.12$. One can see this result is going to the same direction as ours to improve the naive first order estimate $\left(\Sigma_{\pi N} \simeq 26 \mathrm{MeV}\right.$ with $y=0$ ), although they are seemingly different approximation schemes. This observation is totally consistent with that mentioned by Chan and Haymaker for $f_{K} / f_{\pi}[43]$.

Although the $\bar{s} s$ content is smaller than $\bar{u} u$ or $\bar{d} d$ in nucleon, it is not negligible. Furthermore, the non-strange content of $\Omega^{-}$is even larger than the strangeness content of the proton. This is simply because the $u(d)$-quark has a mass (current and constituent) smaller than that of the s-quark, hence gets easily excited.

Here let us give a brief discussion on the convergence radius of chiral perturbation in QCD. The chiral perturbation theory, which assumes the smallness of the current quark masses, is one of the good starting points to study these phenomena as far as $m$ is small enough compared to the typical scale of QCD e.g. $\Lambda_{Q C D} \sim 200 \mathrm{MeV}$. This condition is well satisfied for the $u$ and $d$ quarks since $m_{u, d} \sim O(10) \mathrm{MeV}$, while it is merginal for the $s$ quark $\left(m_{s} \sim 100-200 \mathrm{MeV}\right.$. Therefore it will be important to know the higher order behavior of the expansion by the current quark masses.

So far, two essential features of the higher order chiral perturbation $(\chi \mathrm{P})$ have been observed: (I) Non-analytic terms due to the loops of Nambu-Goldstone (NG) bosons (Li and Pagels), which becomes an important correction to the 1st order result in some cases. (II) The question on the convergence of the perturbation series (Carruthers and Haymaker $(\mathrm{CH})$ ) in the tree level of the meson fields or in the large $N_{c}$ limit. The former is related to the non-analitic terms of the current quark masses such as $m \operatorname{lnm}, \mathrm{m}^{3 / 2} \mathrm{etc}$, while the latter is related to the convergence properties of ther analytic series $\sum_{n} c_{n} m^{n}$. The latter series does not make sense when $m$ is sufficiently large, then the former non-analytic corrections also becomes meaningless in this situation. In the following, I will first discuss an "existence theorem" of such convergence radius of the analytic series in the large $N_{c}$ QCD and then quantitative estimates of the radius are given using effective theories of QCD.

A fundamental quantity in the chirally broken world is the chiral condensate $\langle\bar{q} q\rangle$ which controlls almost all the physics of light hadrons as QCD sum rules tells us. Since there does not appear the non-analuytic terms due to Nambu-Goldstone bosons in the large $N_{c}$ limit of QCD, we can study the convergence propertiy of the following expansion

$$
\sigma(m) \equiv \frac{1}{N_{c}}\langle\bar{q} q\rangle=\sum_{n} C_{n} m^{n}
$$

with $C_{0}$ being the condensate in the chiral limit. $\sigma(m)$ in the large $N_{c}$ limit is determined from the minimization of the effective potential $V(\sigma)$ in the same limit which can be formally written down using the constrainrd path integral (this is known 
to be equaivalent to the usual efective potential through the Legendre transform in the large $N_{c}$ limit);

$$
e^{-V(\sigma)}=\int[d q][d \bar{q}][d A] e^{S_{Q C D}} \delta\left(\sigma-\frac{1}{N_{c}} \bar{q} q\right),
$$

After doing the stationalry phase approximation twice (which is exact in the large $N_{c}$ limit), one gets

$$
V(\sigma)=V_{0}(\sigma)+m \sigma
$$

It is easy to see that the as far as $V_{0}$ has more than two extremum (e.g. the chiral symmetric point and the true minimum), there is always finite convergence radium for the expansion and $m_{\text {con }}$ is determined by the condition $d \sigma(m) / d m=\infty$. This is quite general argument and it can be applied to any model for the dynamical symmetry breaking with explicit breaking term.

Although the proof is quite general and almost independent of the detailed structure of the effective potential in the chiral limit, the actual value of $m_{\text {con }}$ needs explict form of $V_{0}(\sigma)$. To estimate $m_{\text {con }}$, we adopt a simple linear $\sigma$ model and also NJL model. Both are the prototype models for the dynamical breaking of chiral symmetry.

In the effective potential of the linear $\sigma$ model with explicit symmetry breaking

$$
V(\sigma)=\frac{g^{2}}{4}\left(\sigma^{2}-f^{2}\right)^{2}-c \sigma .
$$

The extremum of this potential is determined by a self consistent equation

$$
x\left(x^{2}-1\right)=c / f^{3} g^{2} \equiv a \quad \text { with } \quad x=\sigma / f .
$$

In the chira? limit $(a=0)$, there are three solutions $x=0$ (the symmetric phase) and $x= \pm 1$ (the dynamically broken phases). If one tries to get an exact solution $x(a)(>0)$ by the perturbation around $x(a=0)=1$, the convergence radius of this expansion turns out to be $|a|<a_{c r}=2 / 3 \sqrt{3}$, which is obtained by the point where $d x / d a=\infty$. Ore of the shortcomings of the $\mathrm{CH}$ approach is that the translation of their result in terms of $m_{s}$ is impossible without using the Gell-Mann-Oakes-Renner (GOR) relation whose validity is not obvious by itself in the strange sector. This is the reason why we start with an effective theory with quark fields which allows us to trace the effect of $m_{s}$.

In the NJL model, $\phi=\operatorname{diag}(<\bar{u} u>,<\bar{d} d>,<\bar{s} s>)$ is the quark condensates obtained by $\phi_{j}=-i \operatorname{Tr} S_{F}\left(x ; M_{j}\right)$ with $S_{F}$ being the quark propagator with mass $M_{j}$. This equation can be simply written as

$$
M(F(M)-1)=m,
$$

which corresponds to eq.(3.8) in our simple example. Since physical quantities are always written in terms of $M$, it is sufficient to show that the expansion of $M$ by 
$m$ has finite convergence radius for our purpose. $M=319 \mathrm{MeV} \sim(1 / 3) \Lambda_{x S B}$ in the chiral limit. Then, the con lition $d M / d m=\infty$ and the ga-. equation yeilds

$$
m_{c r}=0.031 \Lambda_{4} \quad\left(0.048 \Lambda_{3}\right),
$$

If one adopts the typical value $\Lambda \simeq \Lambda_{\chi S B}=1 \mathrm{GeV}, m_{c r}$ becomes $30-50 \mathrm{MeV}$. Therefore, the physical $m_{s}$ is out of the convergence radius while $m_{u, d}$ is within the radius.

The above observation explicitely, let us now expand several physical quantities related to pion and kaon by $m_{s}$. In this case, the lst order $\chi \mathrm{P}$ seems to work even around $m_{s} \sim 300 \mathrm{MeV}$. However, this is merely a coincidence, which can be seen by the long wave length approximation of the dispersion relation without $\chi \mathrm{P}$,

$$
m_{p s}^{2} \simeq \frac{G_{p s, q}^{2}}{G_{p s}} \cdot \frac{m_{i}+m_{j}}{M_{i}+M_{j}}
$$

Expanding both the denominator and numerator by $m_{i}$, one recovers the GOR formula. The point is that the large errors due to $\chi \mathrm{P}$ in both denominator $\left(G_{p s, q}^{2}\right)$ and numerator $\left(M_{i}+M_{j}\right)$ cancel out and make seemingly a valid result $m_{p s} \simeq m_{p s}(G O R)$. There is a similar phenomenon in the baryon sector: the Gell-Marn-Oku'vo (GO) relation is reproduced in the 1 st order $\chi \mathrm{P}$, but it does not necessary imply the validity of the expansion by $m_{s}$.

\section{Heavy-quark and gluon contents of baryons}

In the previous section, we have determined the light quark contents of baryons using CQM. Combining this dynamical calculation with the trace anomaly, one can extract tile heavy-quark $h=(c, t, b)$ contents of baryons. For heavy quarks, the first-order quark mass expansion is supposed to be good [31];

$$
\langle\bar{h} h\rangle_{B}=-\frac{1}{12 m_{h}}\left\langle\frac{\alpha_{s}}{\pi} G^{2}\right\rangle_{B}+O\left(\frac{1}{m_{h}^{n \geq 3}}\right),
$$

which shows that the heavy quark content scales as $1 / m_{h}$ in the scalar channel. Inserting this into the trace anomaly equation (1.6), one gets the gluon and heavyquark contents of baryons in terms of only the physical baryon mass and the lightquark contents as

$$
\left\langle\frac{\alpha_{s}}{\pi} G^{2}\right\rangle_{B}=-12 m_{h}\langle\bar{h} h\rangle_{B}=-\frac{8}{9}\left[M_{B}-\sum_{l}^{u, d, s} m_{l}\langle\vec{l} l\rangle_{B}\right] .
$$

This equation was first derived in ref.[14] where light-quark contributions were assumed to be negligible in the r.h.s. of (4.2). Since we have already calculated $\langle\bar{l} l\rangle_{B}$ in the previous section, we need not to make such an assumption. 
For the nucleon, one can further reduce the r.h.s. of eq.(4.2) by using the relation $m_{u}\langle\bar{u} u\rangle_{N}+m_{d}\langle\bar{d} d\rangle_{N} \simeq \Sigma_{\pi N}$

$$
\left\langle\frac{\alpha_{s}}{\pi} G^{2}\right\rangle_{N}=-12 m_{h}\langle\bar{h} h\rangle_{N}=-\frac{8}{9}\left[M_{N}-\Sigma_{\pi N}-m_{s}\langle\bar{s} s\rangle_{N}\right]
$$

in which only the empirically unknown parameter is $\langle\bar{s} s\rangle_{N}$. This equation show that the theoretical values of the gluon and heavy-quark contents crucially depend on the strangeness content of the nucleon because $m_{s}$ is not negligible; for instance, if $y$ were as large as $0.5,\langle\bar{h} h\rangle_{N}$ would change almost by a factor 2 from the value at $y=0$.

In Table 3, we present the gluon, the light-quarks and the charm contents of baryons. (We took $m_{c}(1 \mathrm{GeV})=1.35 \mathrm{GeV}$.) One can see that $\langle\bar{c} c\rangle_{B}$ does not vary so much from one baryon to another and takes about 0.05 . This constancy could be expected since they are pure sea-quarks. On the other hand, the magnitude of the light-quark content depends on the baryons since they contain the valence contributions. If one looks at the fine structure, one finds that the charm in the octet baryons is smaller than that of the decuplet;

$$
\langle\bar{c} c\rangle_{8}=0.04-0.05 \text { and }\langle\bar{c} c\rangle_{10}=0.06-0.07 \text {. }
$$

This is due to OGE; OGE enhances the light-quark contents of the octet and suppresses those in the decuplet as we saw in the previous section. Then the sea contributions have the opposite tendency since their sum is constrained by the sum rule.

In the last column of Table 3 , we show the ratio $P_{c / B} \equiv\langle\bar{c} c\rangle_{B} / \sum_{l}\langle\bar{l}\rangle_{B}$ which is a measure for the "probability" to find charm in low lying baryons. The difference between the octet and the decuplet is further enhanced in this quantity. One of the most interesting quantity related to the experiment is $P_{\mathrm{c} / N}$;

$$
P_{c / N} \equiv \frac{\langle\bar{c} c\rangle_{N}}{\sum_{l}\langle\bar{l} l\rangle_{N}} \simeq 0.5 \%,
$$

which changes $\pm 0.1 \%$ by the change of $\hat{m}$ from $5 \mathrm{MeV}$ to $5.8 \mathrm{MeV}$.

Phenomenologically, the intrinsic charm in the nucleon has been suggested for a description of the charm-production in nuclei in terms of the diffractive dissociation [4]. Furthermore, the analysis of the charm structure function of the proton at large $x$ seems to require an intrinsic charm probability of order 0.3\%.[32] However, non-perturbative estimates of the heavy quark content have been scarce so far; the preceding work that we are aware of is only a calculation using the MIT bag model by Donoghue and Golowich [49]. They obtained the probability of finding charm in the proton to be $\sim 2 \%$. In their case, the charm admixture was solely attributed to the perturtative gluon exchange with a large $\alpha_{s} \simeq 2.2$, so their result should be regarded as a rough estimate. In our case, the result is on the contrary rather stable since the $\bar{c} c$-content is determined by the hadron masses with a small additional contribution from the light quarks as seen in Eq.(4.3). Instead of taking the naive ratio 
$\langle\bar{c} c\rangle_{N} / \sum_{l}\langle\bar{l}\rangle_{N}$ as a "probability" to find charm, one can relate $\langle\bar{c} c\rangle_{N}$ more directly to the intrinsic charm distribution function in the light-cone representation, which again gives the probability $0.5-1 \%$ if one uses eq.(4.5).[50]

$A s$ for $b$ and $t$ quark contents, what one should do is to just multiply the mass suppression factor $m_{c} / m_{b, t}$ to the result for the charm quark, eq.(4.5). Thus, for example,

$$
P_{b / N} \simeq 0.1 \%, \quad P_{t / N} \simeq 0.005 \%,
$$

for $m_{b(t)}=5(130) \mathrm{GeV}$ at $1 \mathrm{GeV}$ scale.

\section{Higgs-baryon coupling constants}

Originally, the Higgs-nucleon coupling strength was derived using the trace anomaly combined with the heavy quark mass expansion by SVZ [14]: once one neglects the light quark contributions, the coupling constant is entirely determined by the heavy sea-quarks $(c, b, t)$. This conclusion has, however, been questioned recently by Cheng[17] and Cheng [18], who pointed out that the strange-quark contribution cannot be neglected if one a.dopts $y \simeq 0.5$, with which the coupling strength is enhanced by a factor $2-3$. In thi: section, we will show that both pictures are not quite right but correspond to two opposite extremes.

The simplest form of the coupling of the scalar Higgs-boson to quarks is written as

$$
\mathcal{L}_{\phi q q}=\sum_{q} m_{q}(\phi / v) \bar{q} q
$$

with $v=\left(\sqrt{2} G_{F}\right)^{-1 / 2}=246 \mathrm{GeV}$ being the symmetry breaking scale of the electroweak theory.

The Higgs-baryon coupling constant is given by the baryon matrix element of the mass term of QCD hamiltonian

$$
\begin{aligned}
v g_{\phi B B} & =\sum_{l}<m_{l} \bar{l} l>_{B}+\sum_{h}<m_{h} \bar{h} h>_{B} \\
& \simeq \frac{2}{9} M_{B}+\frac{7}{9} \sum_{l}<m_{l} \bar{l} l>_{B}
\end{aligned}
$$

for 3 light and 3 heavy quarks. ${ }^{14}$

From Table 3 , we can read the light and heavy quark contributions to $v g_{\phi B B}$. For the nucleon, the light quark contribution reads

$$
<m_{u} \bar{u} u+m_{d} \bar{d} d+m_{s} \bar{s} s>_{N} \simeq 122 \pm 30 \mathrm{MeV} .
$$

\footnotetext{
${ }^{14}$ Here the current quart: masses are the ones which do not include the effect of the anomalous dimension.
} 
We remark that the magnitude of this quantity is correlated with that of $y$ : if $y \simeq 0.47$ were adopted instead of our value $y \simeq 0.12$, the same quantity would become as large as $410 \mathrm{M} \in \mathrm{V}$. The heavy quark content reads

$$
\sum_{h} m_{h}\langle\bar{h} h\rangle_{N} \simeq 182 \mp 7 \mathrm{MeV}
$$

Thus one sees that the light-quark contribution is not so large as Cheng [17] and Cheng [18] claim, but not so small as SVZ [14] does.

The coupling constants $v g_{\phi B B}$ as well as the light and heavy quark contributions to it and their ratio are tabulated in Table 4.

One finds that (i) the flavor mixing in the non-strange baryons generally increases $g_{\phi B B}$ since $\langle\bar{s} s\rangle_{B}$ is not negligible, and (ii) as the number of the valence $s$-quark increases, the Higgs-baryon coupling constant increases (the Higgs- $\Omega$ coupling is the largest) since $m_{s}\langle\bar{s} s\rangle_{B}$ overwhelms $m_{u}\langle\bar{u} u\rangle_{B}$ and $m_{d}\langle\bar{d} d\rangle_{B}$ owing to the large difference in the masses, $m_{s} / \hat{m} \simeq 25$.

Our value for the Higgs-nucleon coupling reads

$$
v g_{\phi N N}=303 \pm 25 \mathrm{MeV}=(0.32 \pm 0.03) M_{N}
$$

which is in the middle of the predictions $v g_{\phi N N}=210 \mathrm{MeV}=0.22 M_{N}(y=0)$ [14] and $v g_{\phi N N}=530 \mathrm{MeV}=0.56 M_{N}(y=0.47)[17,18]$.

\section{Quark- and gluon-contents of pion and kaon}

In this section, we will examine the quark- and gluon-contents of the pion and the kaon using the $\mathrm{FH}$ theorem and the trace anomaly again. In applying the $\mathrm{FH}$ theorem, one needs to get the mass $m_{\pi}$ in terms of the current quark masses $m_{i}$. This is nicely accomplished in the NJL model, where the meson masses are obtained from the dispersion relations the derivatives of which with respect to $m_{i}$ can be performed. Before the direct calculation, let us first start with the Gell-Mann-Oakes-Renner (GOR) relation [58] to get some intuition:

$$
m_{\pi}^{2}=-\frac{\hat{m}}{f_{\pi}^{2}}\langle\bar{u} u+\bar{d} d\rangle_{0}+O\left(\hat{m}^{2}\right)+\cdots .
$$

Here the pion decay constant $f_{\pi}$ and the quark condensates are evaluated in the limit $m_{u, d} \rightarrow 0$ but not necessarily in the limit $m_{s} \rightarrow 0$; i.e., they can be a non-linear function of $m_{s}$. One can easily see this by looking at the derivation of the GOR relation carefully.(See e.g., ref.[59].) We remark that the GOR formula is naturally derived from the dispersion equation in the NJL model [36] as it should be.

The FH theorem applied to pion reads

$$
\frac{\partial m_{\pi}}{\partial m_{i}}=\left\langle\bar{q}_{i} q_{i}\right\rangle_{\pi}=\left\langle\bar{q}_{i} q_{i}\right\rangle_{\pi}^{c} / 2 m_{\pi},
$$


where $\langle\cdot\rangle_{\pi}^{c}$ denotes the matrix element in the covariant normalization $\left\langle p^{\prime} \mid p\right\rangle=$ $2 \omega_{p}(2 \pi)^{3} \delta^{3}\left(\mathbf{p}^{\prime}-\mathbf{p}\right)$. Then inserting the GOR formula (6.1), one gets

$$
\langle\bar{u} u\rangle_{\pi}^{c}=\langle\bar{d} d\rangle_{\pi}^{c}=\frac{m_{\pi}^{2}}{2 \hat{m}}, \quad\langle\bar{s} s\rangle_{\pi}^{c}=m_{\pi}^{2} \frac{\partial}{\partial m_{s}} \ln \left(\left|\langle\bar{u} u\rangle_{0}\right| / f_{\pi}^{2}\right) .
$$

The former relation could be also derived by using the soft pion theorem and the latter shows that $\langle\bar{s} s\rangle_{\pi}^{c}$ vanishes in the chiral limit $m_{u, d} \rightarrow 0$ as the Nambu-Goldstone theorem tells us. $\langle\bar{s} s\rangle_{\pi}^{c}$ in eq.(6.3) can be explicitly calculated with the use of the NJL model.

Once the light quark content of the pion has been given, the trace anomaly tells:

$$
\begin{aligned}
\left\langle\Theta_{\mu}^{\mu}>_{\pi}^{c}\right. & =2 m_{\pi}^{2}, \\
& \simeq m_{u}\langle\bar{u} u\rangle_{\pi}^{c}+m_{d}\langle\bar{d} d\rangle_{\pi}^{c}+m_{s}\langle\bar{s} s\rangle_{\pi}^{c}-\frac{9}{8}\left\langle\frac{\alpha_{s}}{\pi} G^{2}\right\rangle_{\pi}^{c},
\end{aligned}
$$

where we have applied the heavy quark mass expansion in the second equality. Thus using the result eq.(6.3) derived from the GOR formula eq. (6.1), we get the gluon and heavy-quark contents,

$$
\left\langle\frac{\alpha_{s}}{\pi} G^{2}\right\rangle_{\pi}^{c}=-12 m_{h}\langle\bar{h} h\rangle_{\pi}^{c}=-\frac{8}{9}\left[m_{\pi}^{2}-m_{s}\langle\bar{s} s\rangle_{\pi}^{c}\right],
$$

which vanish in the chiral limit. This implies thet only small values are allowed for them even away from the chiral limit, while such a constraint is absent in baryons.

Now, let us calculate the quark contents of the pion by directly differentiating the pion mass obtained in the NJL model without recourse to the GOR relation, especially for the purpose to evaluate the strangeness content of pion. In the NJL model, the meson masses are obtained dynamically from the dispersion equations. The dispersion equation for the pion $\left(\pi^{ \pm}\right)$reads;

$$
0=D_{\pi}^{-1}\left(m_{\pi}^{2}\right) \propto 1+g_{\pi} \Pi_{\pi \pm}\left(m_{\pi}^{2}\right),
$$

where $g_{\pi}=g_{s}+g_{D}\langle\bar{s} s\rangle_{0}$ and $\Pi_{\pi \pm}\left(k^{2}\right)=I_{u}^{P}\left(k^{2}\right)+I_{d}^{P}\left(k^{2}\right)$ with $I_{i}^{P}\left(k^{2}\right)$ being the loop integral in the pseudoscalar channel. Differentiating the dispersion equation, one gets

$$
\begin{aligned}
\langle\bar{u} u\rangle_{\pi^{ \pm}}=\langle\bar{d} d\rangle_{\pi^{ \pm}} & =6.25 \\
\left|\langle\bar{s} s\rangle_{\pi \pm}\right| /\langle\bar{u} u+\bar{d} d\rangle_{\pi^{ \pm}} & <4.5 \times 10^{-4} \\
\langle\bar{c} c\rangle_{\pi^{ \pm}} /\langle\bar{u} u+\bar{d} d\rangle_{\pi^{ \pm}} & =3.0 \times 10^{-4} \\
\left\langle\frac{\alpha_{s}}{\pi} G^{2}\right\rangle_{\pi^{ \pm}} & =-61.3 \mathrm{MeV} .
\end{aligned}
$$

where $\hat{m}=5.5 \mathrm{MeV}$ is used. The $u$ and $d$ contents of pion are quite consistent with the result in the chiral perturbation as given by eq.(6.3)( which gives the value 6.27) as it should be. It is found that the change of them due to the variation of $\hat{m}$ is 
$\pm 10 \%$. Furthermore, the $c$ content and the gluon content is essentially determined by the pion mass itself since $s$ content is small, hence it is quite insensitive to any variation of the model parameters. In contrast, the $s$ content is quite sensitive to the variation of the current mass $\hat{m}$ : by changing $\hat{m}$ from $5.0 \mathrm{MeV}$ to $5.8 \mathrm{MeV}$, it changes even sign but limited to the range in the above formula. Comparing our result with the value obtained in the chiral perturbation theory by Gasser [48], one finds that it is rather smaller than his number $1.5 \times 10^{-3}$. However our value is consistent with the recent result by Donoghue et. al., where the same quantity is quoted as $6 \times 10^{-4} \sim-6 \times 10^{-4} \cdot[60]$

Comparing (6.8) with the results Table 2 and 3 for baryons, one sees that the seaquarks in the pion are order of magnitude smaller than those in baryons, which is due to the Nambu-Goldstone nature of the pion. This may imply that the pion-induced diffractive charm cross section is order of magnitude smaller than the proton-induced cross section, and also can account for the fact that the $\phi$ production in $p p$ reactions is roughly twice as large as that in $\pi^{ \pm} p$ reactions [1].

Similar analysis can be done for kaon also, which results in

$$
\begin{aligned}
\langle\bar{u} u\rangle_{K^{ \pm}} & =1.78, \\
\langle\bar{s} s\rangle_{K^{ \pm}} & =1.83, \\
\langle\bar{d} d\rangle_{K^{ \pm}} /\langle\bar{u} u+\bar{s} s\rangle_{K^{ \pm}} & =8.8 \times 10^{-3}, \\
\langle\bar{c} c\rangle_{K^{ \pm}} /\langle\bar{u} u+\bar{s} s\rangle_{K^{ \pm}} & =3.6 \times 10^{-3}, \\
\left\langle\frac{\alpha_{s}}{\pi} G^{2}\right\rangle_{K^{ \pm}} & =-211 \mathrm{MeV} .
\end{aligned}
$$

The $u$ and $s$ contents of $K^{ \pm}$are almost the same with the prediction of the GOR relation as in the case of the pion and the changes due to the variation of $\hat{m}$ is less than $5 \%$. (Remember that all the parameters including $m_{s}$ are readjusted to fix the physical quantities as mentioned in section 2 . Note also that this does not necessarily means the 1st order chiral perturbation for the kaon is good as is discussed in [42].) The $c$ and gluon contents are quite insensitive to a change of the parameters since the kaon mass is a dominant term (others are less than $1 \%$ ). Furthermore, the $d$ content is also stable for the variation of $\hat{m}$; the changes are only $10 \%$. The $d$ content is also consistent with the value in the chiral perturbation theory up to one loop, $4.4 \times 10^{-3}$ calculated in ref.[48] including the sign. This also confirms the statement we made in section 2 that these approaches go to the same direction although the starting vacuum is different.

\section{Concluding remarks}

The quark contents in the scalar channel are rather special among those in various channels in the sense that chiral syminetry and a sum rule for hadron masses work quite effectively to extract the values. In other channels, one will have to recourse to 
the explicit models of hadrons to calculate the quark content at low energies, hence the results would become rather model-dependent. Nevertheless, it will be interesting to calculate the quark and gluon contents in various channels using effective theories: The calculation with the MIT bag [49] seems to overestimate the charm content of the nucleon because a large $\alpha_{s} \sim 2.2$ was used, as we mentioned. Recently it has been found that the heavy quarks can be incorporated in the Skyrme model[61] in the bound-state approach, which would be one of effective methods to evaluate the heavy quark contents of baryons. 


\begin{tabular}{|c|c|c|}
\hline & Theory & Empirical values \\
\hline \hline$M_{u}\left(M_{s}\right)$ & $335(527)$ & $336(540) \mathrm{MeV}$ \\
$<\bar{u} u>_{N P}$ & $-(245)^{2}$ & $-(225 \pm 25)^{3} \mathrm{MeV}^{3}$ \\
$\left\langle\bar{s} s>_{N P} /<\bar{u} u>_{N P}\right.$ & 0.78 & $0.8 \pm 0.1$ \\
$m_{\pi}\left(m_{K^{*}}\right)$ & $138^{*}\left(496^{*}\right)$ & $138(496) \mathrm{MeV}$ \\
$m_{\eta}\left(m_{\eta^{\prime}}\right)$ & $487\left(958^{*}\right)$ & $549(958) \mathrm{MeV}$ \\
$m_{\sigma}\left(m_{\sigma^{\prime}}\right)$ & $668(1348)$ & $\sim 700(\sim 1400) \mathrm{MeV}$ \\
$\Gamma_{\sigma}=\operatorname{Im} m_{\sigma}$ & $\sim 450$ & $\sim \operatorname{Re} m_{\sigma}$ \\
$f_{\pi}\left(f_{K}\right)$ & $93.0^{*}(97.7)$ & $93(113) \mathrm{MeV}$ \\
$f_{\eta}\left(f_{\eta^{\prime}}\right)$ & $94.3(103.1)$ & $108 \pm 8(100 \pm 3) \mathrm{MeV}$ \\
$\theta_{\eta}\left(\varphi_{\sigma}\right)$ & $-21^{\circ}\left(-6.8^{\circ}\right)$ & $\sim-20^{\circ}(-)$ \\
$G_{\pi q}\left(G_{K_{q}}\right)$ & $3.5(3.6)$ & $\sim 3.5(-)$ \\
$G_{\pi N}\left(G_{\sigma N}\right)$ & $13.4(10.0)$ & $13.4(\sim 10.0)$ \\
$\Sigma_{\pi N}$ & $49 \pm 7$ & $45 \pm 12 \mathrm{MeV}$ \\
\hline
\end{tabular}

Table 1 : Comparison of the theoretical estimates and the empirical values. (See ref.[44, 7] for the source of the empirical values.) * indicates the quantities used as inputs to determine the parameters. $\theta_{\eta}$ denotes the octet-singlet mixing angle of the $\eta-\eta^{\prime}$ system, while $\varphi_{\sigma}$ the mixing angle of the scalar mesons in the quark basis. $\Gamma_{\sigma}$ is the width of the $\sigma$-meson from the $\pi-\pi$ intermediate state. $G_{\pi N}$ and $G_{\sigma N}$ are obtained by the additive quark ansatz.

\begin{tabular}{|l|c|c|c|}
\hline \multicolumn{1}{|c|}{$\mathrm{B}$} & $\langle\bar{u} u\rangle_{B}$ & $\langle\bar{d} d\rangle_{B}$ & $\langle\bar{s} s\rangle_{B}$ \\
\hline \hline $\mathrm{P}(93 \varepsilon)$ & 4.97 & 4.00 & 0.53 \\
$\Lambda^{0}(1115)$ & 3.63 & 3.63 & 1.74 \\
$\Sigma^{+}(1193)$ & 4.19 & 1.13 & 1.95 \\
$\Xi^{0}(1320)$ & 3.21 & 1.12 & 3.10 \\
\hline$\Delta^{+}(1232)$ & 2.70 & 1.73 & 0.26 \\
$\Delta^{++}(1232)$ & 3.66 & 0.76 & 0.26 \\
$\Sigma^{*+}(1385)$ & 2.88 & 0.76 & 1.25 \\
$\Xi^{* 0}(1507)$ & 1.92 & 0.77 & 2.51 \\
$\Omega^{-}(1672)$ & 0.72 & 0.72 & 3.71 \\
\hline
\end{tabular}

Table 2 : Light quark contents in the octet and decuplet baryons. 


\begin{tabular}{|l|c|c|c|c|}
\hline $\mathrm{B}$ & $-\frac{\alpha_{S}}{\mu}\left\langle G^{2}\right\rangle_{B}$ & $\sum_{l}\langle l l\rangle_{B}$ & $\langle\bar{c} c\rangle_{B}$ & $\langle\bar{c} c\rangle_{B} / \sum_{l}\langle l l\rangle_{B}$ \\
\hline \hline $\mathrm{P}(938)$ & 726 & 9.50 & 0.045 & 0.0047 \\
$\Lambda^{0}(1115)$ & 746 & 9.00 & 0.046 & 0.0051 \\
$\Sigma^{+}(1193)$ & 800 & 7.27 & 0.049 & 0.0068 \\
$\Xi^{0}(1320)$ & 778 & 7.43 & 0.048 & 0.0065 \\
\hline$\Delta^{+}(1232)$ & 1042 & 4.69 & 0.064 & 0.0137 \\
$\Delta^{++}(1232)$ & 1042 & 4.69 & 0.064 & 0.0137 \\
$\Sigma^{*+}(1385)$ & 1063 & 4.88 & 0.066 & 0.0134 \\
$\Xi^{* 0}(1507)$ & 1023 & 5.20 & 0.063 & 0.0122 \\
$\Omega^{-}(1672)$ & 1032 & 5.15 & 0.064 & 0.0124 \\
\hline
\end{tabular}

Table 3 : The gluon, light quarks and the charm contents in baryons. The gluon content is written in $\mathrm{MeV}$ units.

\begin{tabular}{|l|c|c|c|c|}
\hline \multicolumn{1}{|c|}{$\mathrm{B}$} & $\sum_{l} m_{l}\langle l l\rangle_{B}$ & $\sum_{h} m_{h}\langle h h\rangle_{B}$ & $l / h$ & $v g_{\phi B B}$ \\
\hline \hline $\mathrm{P}(938)$ & 121 & 182 & 0.67 & 303 \\
$\Lambda^{0}(1115)$ & 276 & 187 & 1.48 & 462 \\
$\Sigma^{+}(1193)$ & 294 & 200 & 1.47 & 494 \\
$\Xi^{0}(1320)$ & 445 & 195 & 2.29 & 639 \\
\hline$\Delta^{+}(1232)$ & 60 & 261 & 0.23 & 320 \\
$\Delta^{++}(1232)$ & 60 & 261 & 0.23 & 320 \\
$\Sigma^{*+}(1385)$ & 189 & 266 & 0.71 & 455 \\
$\Xi^{* 0}(1507)$ & 356 & 256 & 1.39 & 612 \\
$\Omega^{-}(1672)$ & 511 & 258 & 1.98 & 769 \\
\hline
\end{tabular}

Table 4 : The light and heavy quark contributions to the Higgs-baryon coupling constants. The units are $\mathrm{MeV}$ except for the dimensionless quantity $l / h \equiv$ $\sum_{l} m_{l}\langle\bar{l} l\rangle_{B} / \sum_{h} m_{h}\langle\bar{h} h\rangle_{B}$. 


\section{References}

[1] S. Okubo, Prog. Theor. Phys. Suppl. 63 (1978) 1.

[2] T. P. Cheng, Phys. Rev. D13 (1976) 2161; C. A. Dominguez and P. Langacker, Phys. Rev. D24 (1981) 1905; R. L. Jaffe and C. L. Korba, Comm. Nucl. Part. Phys. 17 (1987) 163.

[3] J. Ashman et. al., Nucl. Phys. B328 (1990) 1.

[4] S. J. Brodsky and P. Hoyer, Phys. Rev. Lett. 63 (1989) 1566; S. J. Brodsky, C. Peterson and N. Sakai, Phys. Rev. D23 (1981) 2745.

[5] J. Ellis, E. Gabathuler and M. Karliner, Phys. Lett. B217 (1989) 173; H. J. Lipkin, Phys. Lett. B225 (1989) 287; C. B. Dover and P. M. Fishbane, Phys. Rev. Lett. 62 (1989) 2917, ibid. 64 (1990) 3115.

[6] T. Kunihiro and T. Hatsuda, Phys. Lett. 240B (1990) 209.

[7] T. Hatsuda and T. Kunihiro, Z. Phys. C (1991) in press.

[8] J. Gasser, H. Leutwyler and M. E. Sainio, Phys. Lett. B253 (1991) 252.

[9] J. Gasser and H. Leutwyler, Phys. Rep. 87 (1982) 77.

[10] T. Hatsuda, Nucl. Phys. B329 (1990) 376; Preprint INT-4-90 (1990), (talk presented at $Q C D$ '90, 8-13 July, 1990, Montpellier, France).

[11] T.-P. Cheng and L.-F. Li, Proton Spin Contents: Current Status and Perspectives, CMU-HEP-90-2 (1990). G. Altarelli, Polarized Electroproduction and the Spin of the Quarks inside the Proton, CERN-TH-5675/90 (1990).

[12] D. M. Alde et. al., Phys. Rev. Lett. 66 (1991) 133, 2285.

[13] S. J. Brodsky, Challenges to Quantum Chromodynamics: Anomalous Spin, Heavy Quark and Nuclear Phenomena, SLAC-PUB-5116 (1989).

[14] A. Shifman, A. I. Vainshtein and V. I. Zakharov, Phys. Lett. 78B (1978) 443.

[15] J. C. Collins, A. Duncan and S. D. Joglekar, Phys. Rev. D16 (1977) 438; N. K. Nielsen, Nucl. Phys. B120 (1977) 212.

[16] R. P. Feynman, Phys. Rev. 56 (1939) 340; W. Weisberger, Phys. Rev. D5 (1972) 2600.

[17] T. P. Cheng, Phys. Rev. 38D (1988) 2869.

[18] H. Y. Cheng, Phys. Lett. 219B (1989) 347. 
[19] A. De Rujula, H. Georgi and S. L. Glashow, Phys. Rev. D12 (1975) 149.

[20] N. Isgur and G. Karl, Phys. Rev. D18 (1978) 4187; ibid., D19 (1979) 2653.

[21] Y. Nambu and G. Jona-Lasinio, Ph'jo. Rev. 122 (1961) 345; 124 (1961) 246.

[22] M. Kobayash: and T. Maskawa. Prog. Theor. Phys. 44 (1970) 1422; M. Kobayashi, H. Kondo and T. Maskawa, Prog. Theor. Phys. 45 (1971) 1955.

[23] V. Mirelli and J. Schechter, Phys. Rev. D15 (1977) 1361; J. Kandaswamy, J. Schechter and M. Singer, Phys. Rev. D17 (1978) 1430.

[24] T. Kunihiro, Soryushiron Kenkyu (Studies in Elementary Particle Physics) (Kyto) 77 No.4 (1988) Dl6, (talk presented at the workshop Pion and Quarks in Nuclei, 3-5 November, 1987, Kyoto, Japan).

[25] T. Kunihiro and T. Hatsuda, Phys. Lett. 206B (1988) 385; T. Kunihiro, Prog. Theor. Phys. 80 (1988) 34.

[26] V. Bernard, R. L. Jaffe and U. -G. Meissner, Nucl. Phys. B308 (1988) 753 ; Y. Kohyama, K. Kubodera and M. Takizawa, Phys. Lett. 208B (1988) 165.

[27] H. Reinhardt and R. Alkofer, Phys. Lett. B207 (1988) 482.

[28] J. P. Blaizot, M. Rho and N. N. Scoccola, Phys. Lett. 209B (1988) 271; H. Yabu, Phys. Lett. 218B (1989) 364; B. Kaplan and J. Klebanov, Nucl. Phys. B335 (1990) 45; C. W. Wong, D. Vuorg, and K-C. Chu, Nucl. Phys. A515 (1990) 686 .

[29] R. L. Jaffe, Phys. Rev. D21 (1980) 3215.

[30] A. E. Nelson, Phys. Lett. 221B (1989) 60.

[31] A. I. Vainshtein, V. I. Zakharov and M. A. Shifman, JETP Lett. 22 (1975) 55;

E. Witten, Nucl. Phys. B104 (1976) 445.

[32] E. Hoffmann and R. Moore, Z. Phys. C20 (1983) 71.

[33] T. Goldman and P. W. Haymaker, Phys. Rev. D24 (1981)724,743.

[34] A. Manohar and H. Georgi, Nucl. Phys. B234 (1984) 189

[35] M. K. Volkov, Ann. Phys. (N.Y.) 157 (1984) 282; A. Dhar, R. Shankar and S. R. Wadia, Phys. Rev. D31 (1985) 3256; D. Ebert and H. Reinhart, Nucl. Phys. B271(1986) 188.

[36] T. Hatsuda and T. Kunihiro, Prog. Theor. Phys. 74 (1985) 765. 
[37] T. Kunihiro, Genshikaku Kenkyu (Studies in Nuclear Physics) (Tokyo), 33(1989) 19, Ryukoku-report, RYU-THP-88/1 (lectures presented at the summer school on nuclear, particle and cosmic ray physics, 31 July - 5 August 1988, Iiyama, Japan); T. Hatsuda and T. Kunihiro, Phys. Rep. (to be published).

[38] T. Hatsuda and T. Kunihiro, Phys. Lett. 198B (1987) 126.

[39] G. 't Hooft, Phys. Rep. 142 (1986) 357; M. A. Shifman, A. I. Vainshtein and V. Z. Zakharov, Nucl. Phys. B163 (1980) 46.

[40] G. A. Miller, B. M. K. Nefkens and L. Slaus, Phys. Rep. 194 (1990) 1.

[41] M. Takizawa, K. Tsushima, Y. Kohyama and K. Kubodera, Nucl. Phys. A507 (1990) 611.

[42] T. Hatsuda, Phys. Rev. Lett. 65 (1990) 543.

[43] L. H. Chan and R. W. Haymaker, Phys. Rev. D13 (1976) 1302.

[44] T. Hatsuda and T. Kunihiro, Prog. Theor. Phys. Suppl. 91 (1987) 284.

[45] H. Pagels, Phys. Rep. 16 (1975) 219.

[46] E. V. Shuryak and J. L. Rosner, Phys. Lett. 218B (1989) 72.

[47] N. N. Scoccola, Univ. Regensburg Preprint TPR-89-39 (1989).

[48] J. Gasser, Ann. Phys. (N.Y.) 136 (1981) 62.

[49] J. F. Donoghue and E. Golowich, Phys. Rev. D15 (1977) 3421.

[50] S. J. Brodsky, private communication; S. J. Brodsky and I. Schmidt, Phys. Rev. D43 (1991) 179.

[51] K. Kang and A. R. White, Phys. Rev. D42 (1990) 835.

[52] T. Hatsuda and M. Umezawa, Phys. Lett. B254 (1991) 493.

[53] W. J. Marciano, Phys. Rev. D21 (1980) 2425.

[54] K. Fukazawa, T. Muta, J. Saito, I. Watanabe, M. Yonezawa and M. Inoue, Prog. Theor. Phys. 85 (1991) 111.

[55] L. I. Reinders, H. Rubinstein and S. Yazaki, Phys. Rep. 87 (1982) 77.

[56] G. E. Brown, K. Kubodera and M. Rho, Phys. Lett. 192B (1987) 273.

[57] J. F. Gunion, H. E. Haber, G. Kane and S. Dawson, Higgs Hunter's Guide, (Addison-Wesley, New York, 1990). 
[58] M. Gell-Mann, R. J. Oakes and B. Renner, Phys. Rev. 175 (1968) 2194.

[59] G. A. Christos, Phys. Rep. 116 (1984) 251.

[60] J. F. Donoghue, J. Gasser and H. Leutwyler, Nucl. Phys. B343 (1990) 341.

[61] M. Rho, D. O. Riska and N. N. Scoccola, Phys. Lett. B251 (1990) 597. 


\section{[II] Anomalous Gluon Contents of Nucleon}

\section{Introduction}

The EMC data on the polarized structure function of the proton [1] suggests that the flavor singlet axial charge of the proton is small;

$$
\begin{aligned}
\left\langle J_{\mu 5}^{0}\right\rangle_{N} \equiv\left\langle\sum_{f} \bar{q}_{f} \gamma_{\mu} \gamma_{5} q_{f}\right\rangle_{N} & =g_{A}^{0} \cdot s_{\mu} \\
g_{A}^{0}\left(Q^{2} \simeq 10 \mathrm{GeV}^{2}\right) & =0.12 \pm 0.17
\end{aligned}
$$

with $s_{\mu}=\bar{N} \gamma_{\mu} \gamma_{5} N$. In this chapter I discuss the possible effects which make $g_{A}^{0}$ small at low energy $\left(Q^{2} \simeq 1 \mathrm{GeV}^{2}\right)$. My starting point is the use of the $U_{A}(1)$-anomaly $\partial \cdot J_{5}^{0}=\partial \cdot K+($ symmetry breaking $)$ by which $g_{A}^{0}$ is given as [2]

$$
g_{A}^{0} \cdot \bar{N} i \gamma_{5} N=\frac{\langle\partial \cdot K+s . b .\rangle_{N}}{2 M_{N}}
$$

The attempt to calculate the r.h.s. of (1.2) using the effective Lagrangian was initiated by myself in ref. 3 and subsequently elaborated in ref.4. The result is summarized as the Goldberger-Treiman (GT) type relation in the $U_{A}(1)$ sector

$$
g_{A}^{0}=\sqrt{\frac{3}{2}} \frac{f_{\eta_{0}} g_{\eta_{0} N}}{M_{N}}
$$

where $f_{\eta_{0}}\left(g_{\eta_{0} N}\right)$ is the decay constant (the coupling to the nucleon) of the flavorsinglet pseudoscalar meson $\eta_{0} \sim \bar{u} u+\bar{d} d+\bar{s} s$. From the analysis of the two photon decay of $\eta$ and $\eta^{\prime}$, it is known that $f_{\eta_{0}} \simeq f_{\pi}$. As for $g_{\eta_{0} N}$, the simple counting rule in the quark model gives $g_{\eta_{0} N} \simeq \sqrt{6 / 25} g_{\pi N}$. These relations together with the usual GT relation $f_{\pi} g_{\pi N}=q_{A}^{3} M_{N}$ imply

$$
g_{A}^{0}=\frac{3}{5} g_{A}^{3} \simeq 0.75
$$

which is much larger than the experimental value in (1.1). Since the $Q^{2}$ evolution of $g_{A}^{0}$ is only logarithmic in ile scaling region, there should be something non-perturbative around the scale $Q^{2} \simeq 1 \mathrm{GeV}^{2}$ to reduce $g_{A}^{0}$. In the following, I discuss several possibilities to fill the gap between the theory and the experiment. 


\section{What is $g_{\eta_{0} N}$ ?}

In eq.(1.3), we have not specified the precise meaning of $g_{\eta_{0}} N$. This can be made clear by taking an effective Lagrangian incorporating the nucleon explicitly.[5]

$$
\begin{aligned}
\mathcal{L} & =\mathcal{L}_{\text {inv }}(U, N) \\
& +(\alpha / 2)\left(\partial \cdot K_{\zeta}\right)^{2}-\beta\left(\partial \cdot K_{g}\right) \eta_{0} \\
& +g_{1} \bar{N} \gamma_{\mu} \gamma_{5} N \partial^{\mu} \eta_{0}+g_{2} \bar{N} i \gamma_{5} N\left(\partial \cdot K_{g}\right)
\end{aligned}
$$

where we have taken the chiral limit in the flayor $S U(2)$ for simplicity which does not spoils the general feature. $\partial \cdot K_{g}$, which supplies a mass to $\eta_{0}$, is a ghost contribution saturating the topological susceptibility $\left\langle(\partial \cdot K)^{2}\right\rangle_{Y M}=i \alpha$. Note that our nucleon field $N$ is defined as chiral invariant, therefore $\mathcal{L}$ is $U_{L}(2) \otimes U_{R}(2)$ invariant except for the $\beta$-term which gives the anomaly

$$
\partial^{\mu} J_{\mu 5}^{0}=\partial \cdot K_{g}
$$

By diagonalizing this Lagrangian in the tree level, it is easy to see that the physical $\eta_{0}-N$ coupling constant is written as a combination of $g_{1}$ and $g_{2}$ but $g_{\eta_{0} N}$ appearing in (1.3) is not:

$$
\begin{aligned}
g_{\eta_{0} N}^{p h y s} & =g_{1}+(\alpha / \beta) g_{2} \\
g_{\eta_{0} N} & =g_{1}
\end{aligned}
$$

which implies the ghost giving a mass to $\eta_{0}$ has nothing to do with the smallness of $g_{A}^{0}$. The same result can be ubtained by using the other kind of the effective Lagrangian based on the instanton-induced inter 'ions.[6] Furthermore, (2.3) is quite consistent with a more general argument given i.1 ref.7.

Thus one has to look for the reason why $g_{1}$ is so small (e.g. such as the presence of a term like $g_{1}^{\prime} \bar{N} i \gamma_{5} N \eta_{0}$ which breaks $U_{A}(1)$ invariance explicitly [8]) or the other effect not included in the simple effective Lagrangian (e.g. the effect beyond the tree level). I am going to discuss the latter aspect in some detail in the following.

\section{Form factor of $g_{\eta_{0} N}$ and the pole dominance}

One of the natural questions about the equations (1.3) and (2.3) are (i) the effect of the form factor of $g_{\eta_{0} N}$ and (ii) the validity of the $\eta_{0}$ pole dominance. Let us consider the problem in the same way with the analysis of the $6 \%$ discrepancy of the GT relation. The form factor is written as

$$
g_{p s, N^{\prime}}(t)=g_{p s, N}\left(t=\mu^{2}\right) F(t) \quad\left(p s=\pi, \eta_{0}\right),
$$


with the one subtracted dispersion relation

$$
\operatorname{Re} F(0)=1-\frac{\mu^{2}}{\pi} \int_{t_{0}}^{\infty} \frac{\operatorname{Im} F(t)}{t\left(t-\mu^{2}\right)} d t
$$

which corresponds to the assumption of the unsubtracted dispersion relation for the axial charge. (This assumption is not valid for $g_{A}^{0}$ because the corresponding current is not conserved due to the anomaly. Here we focus our attention only the contribution which is finite in the r.h.s. of eq.(3.2). The high energy contribution which needs one more subtraction in eq.(3.2) should be related to effect I will discuss in 4-3.) In the case of the pion, this variation of $F(t)$ is a few percent which is dominated by the $\sigma-\pi$ and $\rho-\pi$ intermediate states. [9]

On the other hand, since $\mu^{2}\left(=m_{\eta_{0}}^{2}\right)$ is large, $F(t)$ might have large variation and might give $F(0) \sim 0$ in our case. The possible low mass intermediate states are (i) $\sigma-\eta_{8}$ and $\delta-\pi$, (ii) $\rho-\pi$ and $3 \pi$, and (iii) $\eta_{8}-\pi-\pi$. By the explicit calculation using (3.2), one easily finds that (i) gives at most $5 \%$ reduction of $F$. (ii) occurs only through the isospin breaking therefore small and (iii) occurs through the $S U_{f}(3)$ breaking and/or the higher order of the large $N_{c}$. In fact the reason why the hadronic width of $\eta^{\prime}$ is so small (comparable to the electromagnetic width) is the small effect from (ii) and (iii).

Although the explicit calculation for (iii) to confirm this using the detailed dispersion technique such as given in ref.[11], it is hard to imagine they have $100 \%$ effect. This is supported also by the validity of the rho-dominance in various process even if there is the the uncorrelated $2 \pi$ effect which is not suppressed by any symmetry in this case.

Thus as far as we limit ourselves to the low energy dynamics described by (2.1), the pole dominance seems to be valid.

\section{Residual effects of $\partial \cdot K$ ?}

There is a possibility that we have neglected something important for $g_{A}^{0}$ when we wrote down the effective Lagrangian. Especially, we took into account only the ghost contribution as an effect of the anomaly in sec.2, or schematically

$$
\partial \cdot K=\partial \cdot K_{g}^{\prime}+: \partial \cdot K^{\prime}
$$

where $: \partial \cdot K:$ denotes the residual effect that might contribute to reduce $g_{A}^{0}$. One can imagine several candidates for $: \partial \cdot K$ : e.g. the instanton effect, the contribution from the real excitations such as the pseudoscalar glueballs, and the short or medium

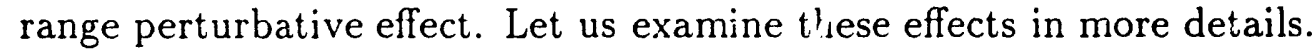

\section{4-1. Instanton Effect}


As is well known, the instanton produces the effective meson vertex [12] $\partial$. $K^{\prime}=\left(\kappa / f_{\pi}\right) \operatorname{Im} \operatorname{det} M$ with $M$ being the $N_{f} \times N_{f}$ meson matrix. In the case of $S U_{f}(3)$, the explicit form reads

$$
\begin{aligned}
\partial \cdot K^{\prime} & =\left(\eta_{0}-\text { pole }\right) \\
& -\frac{\kappa}{\sqrt{2}}\left(2 \sigma_{0} \eta_{0}-\sigma_{8} \eta_{8}-\vec{\delta} \cdot \vec{\pi}\right)+\cdots,
\end{aligned}
$$

where $\kappa\left(=2 \sqrt{2}\left(m_{\eta^{\prime}}^{2}+m_{\eta}^{2}-2 m_{k}^{2}\right)\right)$ is a coefficient determined to reproduce the observed $\eta^{\prime}$ mass. If one takes the nucleon matrix element of $\partial \cdot K$, the 1st term in (4.2) gives a pole contribution while the 2 nd term gives one-loop but finite contribution. The latter replaces $g_{\eta_{0} N}$ in eq.(1.3) by the effective vertex

$$
g_{\eta_{0} N} \rightarrow g_{\eta_{0} N}^{e f f}=g_{\eta_{0} N}^{\text {pole }}\left[1-\kappa \cdot C\left(m_{\sigma}\right)\right],
$$

where $C$ denotes the loop-integral and is essentially a function of the mass of the isosinglet scalar meson $(\sigma)$ summarizing the correlated $2 \pi$ effect. Taking the empirical value of the $\sigma-N$ coupling constant $\left(g_{\sigma N} \simeq 10\right)$, the $\eta-\eta^{\prime}$ mixing angle $\left(\theta \simeq-19^{\circ}\right)$ and the $\delta-N$ coupling constant determined by the $\pi-N$ sigma-term

$$
\frac{g_{\delta N}}{g_{\sigma N}} \simeq \frac{\langle\bar{u} u-\bar{d} d\rangle_{N}}{\langle\bar{u} u+\bar{d} d\rangle_{N}} \simeq \frac{M_{\Xi}-M_{\Sigma}}{\left(m_{s} / \hat{m}-1\right) \Sigma_{\pi N}},
$$

one gets $1-\kappa \cdot C$ as a function of $m_{\sigma}$ as follows:

\begin{tabular}{|c|c|c|c|}
\hline$m_{\sigma}(\mathrm{MeV})$ & 300 & 550 & 750 \\
\hline $1-\kappa \cdot C$ & 0.54 & 0.83 & 0.99 \\
\hline
\end{tabular}

If we take the optimal value of $m_{\sigma}$ deduced from the nuclear force $(\sim 550 \mathrm{MeV})$, one can expect $O(20 \%)$ reduction of $g_{A}^{0}$. However the result is quite sensitive to the change of the parameters $\left(g_{\sigma N}, g_{\delta N}, \theta\right.$ etc). This which is because there is always the cancellation between the singlet piece $\left(+2 \sigma_{0} \eta_{0}\right)$ and the octet-triplet piece $\left(-\sigma_{8} \eta_{8}-\delta \pi\right)$ niece.

\section{4-2. Effect of $0^{-}$glueballs}

There is a strong evidence that $\iota(1430)$ has a sizeable glueball component from the detailed analysis of the radiative decay of $J / \psi$ into pseudoscalar mesons $\left(\eta, \eta^{\prime}\right.$ and ८).[13] This implies that the low lying pseudoscalar glueball $\phi$ can be a main residual effect in (4.1)

$$
\partial \cdot K=\partial \cdot K_{g}+c_{2} \phi
$$

with

$$
\left\langle\left(\partial \cdot K^{\prime}\right)^{2}\right\rangle_{Y^{\prime} M}=i\left(\alpha-c_{2}^{2} / m_{\phi}^{2}\right) .
$$


Furthermore, to give a correct $U_{A}(1)$ anomaly in the effective Lagrangian, $\eta_{0}$ has to couple only with the sum $\partial \cdot K$.[14] Thus the effective Lagrangian can be written as

$$
\begin{aligned}
\mathcal{L} & =\mathcal{L}_{\text {inv }}(U, N) \\
& +\frac{\alpha}{2}\left(\partial \cdot K_{g}\right)^{2}+\frac{1}{2}\left(\partial_{\mu} \phi\right)^{2}-\frac{1}{2} m_{\phi}^{2} \phi^{2} \\
& +\beta\left(\partial \cdot K_{g}+c_{2} \phi\right) \eta_{0} \\
& +g_{1} \bar{N} \gamma_{\mu} \gamma_{5} N \partial^{\mu} \eta_{0} \\
& +g_{2} \bar{N} i \gamma_{5} N\left(\partial \cdot K_{g}+c_{2} \phi\right),
\end{aligned}
$$

The $\beta$ term in the r.h.s. tells us that there is a mixing among $\eta_{0}, \partial \cdot K_{g}$ and $\phi$ which is controlled by the strength of $\beta$ and $c_{2}$.

At first glance, it seems (4.5) is a promising clue to solve the problem of small $g_{A}^{0}$. In fact, if one takes the nucleon matrix element of (4.5), one gets

$$
g_{A}^{0} \propto\left\langle\partial K_{g}\right\rangle_{N}+\left\langle c_{2} \phi\right\rangle_{N}
$$

If the 2 nd term in the r.h.s. of (4.8) has negative contribution, it tends to reduce the 1 st term which gives (1.3) in the absence of $c_{2}$.

However this is not the case. Once one treats the eq.(4.5) and the nuxing among $\eta_{0}, \partial \cdot K_{g}$ and $\phi$ simultaneously (which is a requirement from the anomaly), one finds that there is no effect of the glueballs as well as the ghost to $g_{A}^{0}$. Namely, the relation $g_{\eta_{0} N}=g_{1}$ still holds and $g_{A}^{0}$ is completely independent of $c_{2}, m_{\phi}$ etc. (The particle-mixing effect cancels out the contribution $c_{2}\langle\phi\rangle_{N}$.)

Thus, as far as one takes the pole approximation, the ghost and glueballs do not do anything to reduce the value of $g_{A}^{0}$.

\section{4-3. Perturbative effect of $\partial \cdot K$}

We have been concentrating only on the long wave length excitations coupled to the operator $\partial \cdot K$. However, a rather short-range effect with explicit gluon degrees of freedom might have sizeable effect, or in other words

$$
\partial \cdot K^{\prime}=\left(\partial \cdot K^{\prime}\right)_{L R}+\left(\partial \cdot K^{\prime}\right)_{S R} .
$$

This kind of decomposition in the coordinate space is nicely accomplished by adopting the hybrid bag model.[15] $L R$ (the long range part) is taken into account as the mason clouds outside the bag while $S R$ (the short range part) is calculated using the cavity perturbation theory where the non-perturbative effect is taken into account by using the wave function of quarks and gluons confined in the bag. If one takes only the valence quarks, it can be shown that

$$
\left\langle\left(\partial \cdot K^{\prime}\right)_{S R}\right\rangle_{N}^{v a l} \propto \vec{E}_{c l}^{a} \cdot \vec{B}_{c l}^{a}
$$

where $\vec{E}_{c l}^{a}\left(\vec{B}_{c l}^{a}\right)$ is the classical color electric (magnetic) field inside the bag induced by the valence quarks. Because of the color-singletness of the nucleon, the above 
quantity is zero. However, once one takes into account the excitation of the quarks to non-valence orbits, the above relation does not hold any more. This kind of quantum calculation for the nucleon matrix element of $\left(\partial \cdot K^{\prime}\right)_{S R}$ inside the cavity is under way.[16]

\section{Concluding remarks}

I have shown that there are no effects of ghost and glueballs in the pole approximation. In the loop level, there are several possible candidates to reduce $g_{A}^{0}$ such as the instanton-induced interaction and the short range gluonic interaction. However, a definite and clear reason for the small $g_{A}^{0}$ is still missing. 


\section{References}

[1] J. Ashman et. al., Nucl. Phys. B328 (1989) 1.

[2] T.P. Cheng and L.-F. Li, Phys. Rev. Lett. 62 (1989) 1441.

[3] T. Hatsuda, Nucl. Phys. B329 (1990) 376.

[4] G. Veneziano, Mod. Phys. Lett. A4 (1989) 1605.

[5] T. Hatsuda and T. Kunihiro, unpublished (1990).

[6] T. Hatsuda and T. Kunihiro, SUNY-preprint NTG-89-49 (1989).

[7] G.M. Shore and G. Veneziano, Phys. Lett. 244B (1990) 75.

[8] T. Kunihiro, private communication.

G. Veneziano, private communication.

[9] H.F. Jones and M.D. Suauron, Phys. Rev. D11 (1975) 174.

[î̂] H. Fritzsch, preprint CERN-TH.5676/90 March (1990).

[11] S. Furuichi and K. Watanabe, Prog. Theor. Phys. 83 (1990) 565.

[12] G. 'tHooft, Phys. Rep. 142 (1986) 357.

[13] N. Morishita, I. Kitamura and T. Teshima, preprint CU-90-11 May (1990).

[14] C. Rozenzweig, A. Salomone and J. Schechter, Phys. Rev. D24 (1981) 2545.

[15] T. Hatsuda and I. Zahed, Phys. Lett. 221B (1989) 173.

B.-Y. Park, V. Vento, M. Rho and G. E. Brown, Nucl. Phys. A504 (1989) 829.

[16] A. Hosaka and T. Hatsuda, under investigation. 


\section{[III] Hot and Dense QCD}

\section{Introduction}

The QCD coupling constant $g(\mu)$ decreases logarithmically as one increases the typical scale of the process because of the asymptotic freedom. In the one-loop level,

$$
\frac{g^{2}(\mu)}{4 \pi}=\frac{12 \pi}{\left(33-2 N_{f}\right) \ln \left(\mu^{2} / \Lambda^{2}\right)},
$$

where $N_{f}$ is the active number of flavors and $\mu$ is the renormalization point. In the deep inelastic lepton-hadron scattering, $\mu$ is chosen to be the 4-momentum transfer $\sqrt{Q^{2}}$, while in extremely hot and dense QCD medium, $\mu$ will be proportional to $T$ (temperature) or chemical potential of the system. The latter aspect is a reason why we expect the existence of the quark-gluon plasma i.e. the weakly interacting system of quarks and gluons at high $T$ and/or high baryon density $\rho .[1]$

On the other hand, at low $T-\rho$, long range fluctuations of QCD is well described by effective field theories of mesons and baryons. Therefore, an unified description of the equilibrium system from low $T-\rho$ to high $T-\rho$ is one of the fundamental problems in QCD. So far, there have been number of attempts on this issue using (i) the perturbative QCD and its improved versior in high $T-\rho$ region, (ii) the lattice QCD for $T \sim T_{c}$ with $\rho=0$, and (iii) effective theories and QCD sum rules in low $T-\rho$ region. A tentative goal of these studies is to cover the phase diagram in $(T, \rho)$ plane by extending the applicable range of (i), (ii) and (iii), which is a kind of patch work. Both theoretical developments and experimental information are inevitable for this task. In this chapter, I am going to discuss some of the recent theoretical progress in (i), (ii) and (iii).

\section{$2 \mathrm{QCD}$ at high temperature $\left(T>T_{c}, \rho=0\right)$}

\subsection{Three scales at $T>T_{c}$}

It is now well known that there exists non-perturbative physics in the infrared scale even if $T$ is considerably larger than $T_{c}$. [2] This aspect is summarized as the following three scale structure:

$$
g^{2} T<g T<T,
$$

with $g$ being the running coupling constant at finite $T . g T$ is a scale of the electric screening mass of gluons $\left(m_{e l}=g T \sqrt{N_{c} / 3+N_{f} / 6}\right)$ and $g^{2} T$ is supposed to be related 
to the magnetic screening mass. As far as one stays in low orders of $g$, we have a consistent perturbation theory for static and dynamical properties of the system. Perturbative calculation of the energy density, pressure etc [3] and also the damping rate of the color plasmon etc [4] are the typical examples. On the other hand, once one tries to go to higher orders or to look at the system by a probe of energy scale $O\left(g^{2} T\right)$, the perturbation theory starts to break down, e.g. at $O\left(g^{6}\right)$ for the free energy and at $O\left(g^{4}\right)$ for the magnetic mass.[2] In this case, one has to sum up infinite numbers of diagrams to get physical results, which is certainly a non-perturbative task. $g^{2} T$ is also related to the coupling constant of 3D QCD as high $T$ limit of $4 D$ QCD; the non-perturbative nature of $g^{2} T$ is also inferred from the confining nature of 3D QCD.[5]

As an intuitive tool to understand the coexistence of non-perturbative (NP) and perturbative (P) physics at high $T$, let us introduce a scale $K$ in momentum space which separates the NP and P. Since the NP region is limited in a finite volume in momentum space, it will not affect the bulk properties of the system for high enough $T$ where the typical frequency of quarks and gluons is (a few) $\times T \gg K$. However, if one probes low frequency region or decreases $T$ toward $T_{c}$, effect of the NP region emerges. This feature is actually seen in the lattice QCD simulations of the energy density $\mathcal{E}$, pressure $\mathcal{P}$ and the hadronic screening mass in the scalar-pseudoscalar channel, which we will see in the following subsections.

\subsection{Energy density and pressure on the lattice}

A recent pure gauge $\left(S U_{c}(3)\right)$ simulation of $\mathcal{E}$ and $\mathcal{P}$ on the $16^{3} \times 4$ lattice[6] shows the following behavior:

Stefan-Boltzmann limit (perturbative physics): At enough high $T$, it seems that both $\overline{\mathcal{E}}$ and $\mathcal{P}$ approach the Stefan-Boltzmann (SB) limit (with $g^{2}$ corrections), which means weakly interacting massless gluons dominate the bulk properties of the system. Cutoff scale $K$ (non-perturbative physics): As $T$ approaches $T_{c}$, a sizable discrepancy between $\mathcal{E}$ and $3 \mathcal{P}$ emerges; in particular $\mathcal{P}$ is much smaller than its $\mathrm{SB}$ value. This discrepancy can be seen even up to $\sim 2 T_{c}$ (note that the difference of $\mathcal{E}$ and $3 \mathcal{P}$ starts from $g^{4}$ in perturbation theory)[3], which suggests that the system is not at all the free gluon gas up to $\sim 2 T_{c}$.

Now what is the physics behind this NP aspect? To get a rough idea, let us take a "cutoff model" to simulate the NP physics, $[9,10]$ where a cutoff $K(T)$ is introduced as a scale separating the gluon momentum in the partition function

$$
\ln Z_{Q C D}(T, V)=\ln Z_{\mathrm{NP}}(|\vec{p}|<K)+\ln Z_{\mathrm{P}}(|\vec{p}|>K) \text {. }
$$

The perturbation theory is supposed to work above $K(T)$, while only massive (probably color singlet) modes exist below $K(T)$. To determine the absolute value and $T$ dependence of $K$, lattice data of $\mathcal{E}$ and $\mathcal{P}$ (after the extrapolation to the continuum limit) in $S U_{c}(2)$ gauge theory are used in ref.[9];

$$
K^{\prime}=2.9 T_{c}\left(\frac{T_{c}}{T-T_{c}}\right)^{0.3}
$$


$K$ is found to be as large as $3 T_{c}$ at $T \simeq 2 T_{c}$. Furthermore, by using eq.(4), one can simultaneously fit the color averaged potential between heavy quarks at finite $T$.[10] The simple Debye screening fails to fit the lattice data at large separation, while the cutoff model fits the data very well without introducing extra free parameters. Physical reason of this excellent agreement is rather simple: because of the low momentum cutoff of the gluon propagator, Debye mass gets reduced near $T_{c}$ and hence the potential becomes long range.

Although the above cutoff model seems to work well phenomenologically, it is not an unique way to reproduce the discrepancy between $\mathcal{E}$ and $3 \mathcal{P}$. In fact, trace anomaly in QCD tells us that $\mathcal{E}-3 \mathcal{P}$ is nothing but the gluon condensate

$$
\left\langle\Theta_{\mu}^{\mu}\right\rangle_{T}=\mathcal{E}-3 \mathcal{P}=\frac{\beta}{4 \alpha_{s}}\left\langle G^{2}\right\rangle_{T}
$$

Therefore, one can interpret the non-zero value of $\mathcal{E}-3 \mathcal{P}$ above $T_{c}$ by $\left\langle G^{2}\right\rangle_{T}$.

This has been actually known even before the observation of $\mathcal{E} \neq 3 \mathcal{P}$. Di Giacommo et al.[11] had direct calculation of $\left\langle G^{2}\right\rangle_{T}$ on the lattice and it was subsequently elaborated by Su H. Lee [12]. The result of the latter work tells us that $\left\langle E^{2}\right\rangle_{T \simeq 1.5 T_{c}} \simeq$

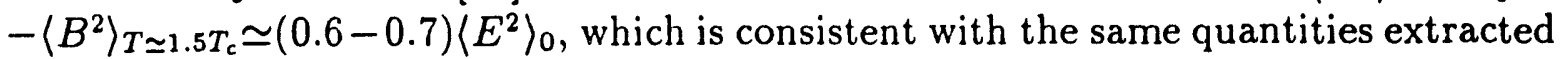
from the Monte Carlo data of $\mathcal{E}$ and $\mathcal{P}$.[13]

Whether the non-vanishing gluon condensate can reproduce $V(r, T)$ or not is an interesting question. One can study this problem by generalizing Shifman's analysis relating the Wilson loop and gluon condensate to finite temperature. This is now under investigation by $\mathrm{Su} \mathrm{H}$. Lee and myself [14].

Now how to check the NP phenomena above $T_{c}$ in experiment? One of the interesting ideas by Gorenstein et al.[10] is to look at the production of $e^{+} e^{-}$pair of the low invariant mass region $\left(M_{e^{+} e^{-}}<2 m_{\pi}\right)$ in the relativistic heavy ion collisions. Due to the cutoff $K$ in the quark spectrum, the momentum distribution of the $e^{+} e^{-}$ yield has a sudden drop below $2 K$. (The kinematical region $M_{e^{+} e^{-}}<2 m_{\pi}$ is needed to get rid of the large hadronic background $\pi^{+} \pi^{-} \rightarrow e^{+} e^{-}$.)

2.3. Hadronic Screening mass

Another interesting quantity, which can be measured on the lattice and shows both $\mathbf{P}$ and NP aspects, is the hadronic screening mass defined by the asymptotic behavior of the correlator for large $|z|$,

$$
\int d x d y d \tau\langle A(\vec{x}, \tau) B(\overrightarrow{0}, 0)\rangle_{T} \rightarrow \exp [-\mu(T) \mid z \|]
$$

with $A$ and $B$ being spacially separated operators having quantum numbers of hadrons. The lattice calculation of this quantity was done by DeTar and Kogut[15] for the first time and subsequently studied by other groups.[16]

Eletskii-Ioffe limit (perturbative physics): Above the critical temperature of chiral transition, one finds (i) there is a parity doubling $\left(\pi-\sigma, \rho-a_{1}\right.$ and $\left.N_{+}-N_{-}\right)$, which 
is a clear signal of the restoration of chiral symmetry, and (ii) screening masses for mesons approach $2 \pi T$ and those for baryons approach $3 \pi T$. This is an evidence of the existence of massless free quarks above $T_{c}$. In fact, as was pointed out by Eletskii and Ioffe (EL),[17] the hadronic screening masses at high $T$ are essentially controlled by the lowest Matsubara frequency of quarks $(\pi T)$. This is just a kinematical and channel independent statement, and lattice data show that this is actually satisfied even near $T_{c}$ (except for the $\pi-\sigma$ channel). Recent data on larger lattices also confirm this approach to the EL limit.[18]

In the above argument, we have neglected $O\left(g^{2}\right)$ corrections to the EL limit, although $g^{2}$ on the lattice is not negligibly small. Therefore, the calculation of the perturbative correction to hadronic screening masses in the continuum and lattice QCD at finite $T$ is important. Such perturbative correction certainly introduces the channel dependence of $\mu(T)$, which might explain the different behavior of $\mu(T)$ between $\rho-a_{1}$ and $\pi-\sigma$. (Note that the spin-spin force due to the one gluon exchange is attractive (repulsive) for the pion (the rho-meson).) This is currently under investigation by $Y$. Koike and myself.[19]

Soft modes (non-perturbative physics): The screening mass in the $\pi-\sigma$ channel is considerably smaller than the EL limit $(2 \pi T)$. This is also seen in another calculation by Gottlieb et al.[20]. This suggests that there remains attractive residual interaction in this channel above $T_{c}$, which may be due to the perturbative interaction explained above or due to the other non-perturbative effect. The latter possibility has been discussed by $T$. Kunihiro and myself even before the lattice calculation[21], where an effective interaction through the dilute instanton gas above $T_{c}$ is considered as an origin of the attraction. (Note that the screening mass and the real excitation mass do not have much difference in the $\pi-\sigma$ channel.[22])

We have shown that this $\pi-\sigma$ modes above $T_{c}$ have good properties as quasiparticles (low mass and narrow width)[21], which led us to call them "soft modes" following the similar excitations in many body physics such as paramagnon and paring vibration. Effect of such light color singlet modes on the static and dynamical properties of the quark-gluon plasma is an interesting future problem.

Not only the hadronic screening mass but also the quark-number susceptibility could be a measure of the $\mathbf{P}$ and NP physics at finite $T$, which is extensively discussed by T. Kunihiro.[23]

\section{Physics at $T \leq T_{c}$}

\subsection{Order of the phase transition}

The determination of the order of the QCD phase transition at finite $T$ is one of the central issues of the recent lattice QCD study.[24] As for the pure gauge system 
without dynamical fermion $\left(m_{q}=\infty\right)$, the center symmetry $\left(Z(3)\right.$ in $S U_{c}(3)$ case) controls the confinement-deconfinement phase transition. The effective $Z(3)$ spin model predicts the 1st order transition and the lattice studies with finite scaling analyses support this feature.[25] Although it is of 1st order, the transition is much weaker than that seen before on the smaller lattice. Once one introduces dynamical fermions, $Z(3)$ symmetry is explicitly broken. However, as far as $m_{q}$ is large enough, one can still study the phase transition based on this approximate symmetry.

On the other hand, in the opposite limit where $m_{q}$ is zero, chiral symmetry instead of $Z(3)$ symmetry takes place to control the phase transition with $\langle\bar{q} q\rangle_{T}$ as an order parameter. For finite quark masses $\left(m_{u, d}=O(10 \mathrm{MeV})\right.$ and $\left.m_{s}=O(200 \mathrm{MeV})\right)$, chiral symmetry is explicitly broken again, but one can still study the phase transition based on the approximate chiral symmetry.

Recent lattice data[26] shows, although the chiral transition near $m_{q}=0$ seems to be 1st order, the phase transition is not observed for the realistic values of $m_{u, d, s}$. There are several predictions for the order of the chiral transition on the basis of the $\sigma$-models and instanton liquid model. [27,28] While the prediction of the order and $m_{q}$ dependence of the transition is a difficult task, the global behavior of $\langle\bar{q} q\rangle_{T}$ is rather easy to access and is actually more important in relation to the experimental observables.

\subsection{Global behavior of $\langle\bar{q} q\rangle_{T}$}

The light quark condensate changes rapidly around $T_{c} \simeq 200 \mathrm{MeV}$ while the heavy one has only smooth change across this temperature, which is a result of the NJL model[29] and is consistent with the similar behavior observed on the lattice.[31] The result of the chira! Lagrangian approach, where the thermal pions and heavy mesons are responsible for the restoration of chiral symmetry, gives also a similar result.[32]

As was pointed out by Cleymans et al.[33], $T_{c}$ in any of these approaches is predicted as

$$
T_{\mathrm{c}} \simeq 2 f_{\pi} \sqrt{3 / N_{c}} \sim 2 f_{\pi} \sqrt{3 N_{f} /\left(N_{f}^{2}-1\right)} \simeq 200 \mathrm{MeV},
$$

where the 1st estimate is from the dynamical models such as the ladder QCD and the NJL model, while the 2nd estimate is from the pion gas model without interactions. The two different approaches (quark loop vs meson loop) giving the similar result suggest a quark - hadron duality in the estimate of $T_{c}$. The mismatching of $N_{c}$ and $N_{f}$ dependence in (3.1) would be resolved if one takes into account the higher loops in the quark side (to get the $N_{f}$ dependence) and the summation of higher-meson contributions in the hadron side.

A message from the above is that $\langle\bar{q} q\rangle_{T}$ decreases slowly (proportional to $T^{2}$ ) at low $T$ and changes rapidly near $T_{c} \simeq 2 f_{\pi}$. Associated with this change of the ground state characterized by $\langle\bar{q} q\rangle_{T}$, one can expect the changes of the elementary modes of excitations (mesons and baryons) at finite $T$, which is actually well-known phenomena 
in condensed matter and nuclear physics. Furthermore, the decays of such hadrons in medium can be used as a thermometer of the hot and/or dense matter.

\subsection{Hadrons at finite $T$}

Serious attempt to calculate the hadron properties at finite $T$ has been initiated by the works of Pisarski,[34] Bochkarev-Shaposhnikov,[35] Hatsuda-Kunihiro,[21] Hashimoto-Miyamura-Hirose-Kanki[36] and Matsui-Satz[37] and it is now extensively examined by numbers of authors. In relation to the restoration of chiral symmetry, the low lying hadrons made of $u$ and $d$ quarks (such as $\pi, \sigma, \rho, A_{1}, \omega, N, \Delta \cdots$ ) are particularly interesting since their properties are mostly determined by the quark condensate in the medium $\langle\bar{q} q\rangle_{T}$ as QCD sum rules tells us. The following is a short summary of our current understanding of such light hadrons in medium.[38]

(1) Weak decrease of the hadron masses: In the vacuum, it is well-known that masses of light hadrons are essentially determined by the quark condensate (e.g. $M_{N} \simeq$ $\langle\bar{q} q\rangle_{0}^{1 / 3}, M_{\rho}=\langle\bar{q} q\rangle_{0}^{1 / 3}$ etc).[39] This is true even at finite $T$ where the main effect of $T$ comes from the non-perturbative condensate $\langle\bar{q} q\rangle_{T}$ and not from the $T$-dependence of the Wilson coefficients or from the scattering with thermal pions.[40] Thus in general

$$
M^{*} \sim-\langle\bar{q} q\rangle_{T}^{1 / 3} \sim\left(1-T^{2} / T_{c}^{2}\right)^{1 / 6}
$$

where we have neglected a small effect of gluon condensate and assumed a mean field behavior $\langle\bar{q} q\rangle_{T} /\langle\bar{q} q\rangle_{0}=\sqrt{1-T^{2} / T_{c}^{2}}$ with $T_{c} \simeq 2 f_{\pi}$. Just because of the dimensional reason, $M^{*}$ has rather weak $T$ dependence and decreases rapidly only above $\sim 0.8 T_{c}$. Such a constancy of the hadron masses below $0.8 T_{c}$ is also reproduced in the chiral models.[41]

(2) Super weak $T$ dependence of NG-boson masses: Since the chiral symmetry is broken below $T_{c}$, there still exist Nambu-Goldstone (NG) bosons[42] and the Gell-MannOkes-Renner relation holds for the real part of the masses[21, 30]

$$
M_{N G}^{*} \sim \sqrt{-m\langle\bar{q} q\rangle_{T} / f_{\pi}^{* 2}}
$$

This shows that, because of the extra mass scale $m, T$ dependence of $M_{N G}^{*}$ is weaker than $M^{*}$ in (3.2). (Note that $f_{\pi}^{*}(T)$ decreases as $T$ increases, [43] which balances the decrease of $\langle\bar{q} q\rangle_{T}$ and makes $M_{N G}^{*}$ almost constant.) The fate of $M_{N G}^{*}$ near $T_{c}$ is not clearly known yet; for example, the NJL model predicts that it is almost constant or slightly increases near $T_{c}[21,22]$, while a simple ansatz $f_{\pi}^{*} \sim\langle\bar{q} q\rangle_{T}^{1 / 3}$ suggests a weak decrease $M_{N G}^{*} \sim\left(1-T^{2} / T_{c}^{2}\right)^{1 / 12}$. The lattice data for the pion screening mass seem to support the first possibility.

(3) Decoupling of hadrons: The width of the scalar and vector mesons and the coupling among hadrons tend to decrease due to the thermal effect, which is a general prediction of the NJL model and similar dynamical models. The decrease of $\rho$ and $\sigma$ width occurs mostly due to the suppression of the decay phase space to $2 \pi$, which dominates over broadening of the width due to the induced emission. Therefore, in a gedanken experiment, the $\rho$ peak reconstructed from $2 \pi$ or lepton pairs moves to 
lower invariant mass region and becomes sharp, while $\sigma$ peak in $2 \pi$, which is hard to see due to its large width at $T=0$, starts to emerge as $T$ increases. In the real experiment of the heavy ion collisions, the detection of lepton pairs (the $2 \pi$ correlation analysis) for the former (latter) is a way to see the effect.[21, 34, 44] As for the interaction between hadrons, its weakening is understood as the melting of the n-point hadron vertices, which can be checked explicitly by making low $T$ and high $T$ expansions of the thermal quark loops in the NJL model. Model independent proof of this nature is needed to confirm the result. There are exceptions of this decoupling because of kinematical reasons; An example is the enhancement of the $\pi^{0} \rightarrow 2 \gamma$ decay rate at finite $T$ where an extra mass scale $m$ plays a significant role.[45]

(4) $U_{A}(1)$ restoration: The nature of the restoration of $U_{A}(1)$ symmetry, which is broken by the axial anomaly at $T=0$, is still an open problem. This is related to the $T$ dependence of the topological susceptibility $\chi_{t}$ and also the $\eta^{\prime}$-meson mass. In the framework of the effective model, there have been several studies.[46, 22]

As one goes to higher orders by taking into account the hadron interactions, the basic aspect discussed above will be modified more or less depending on the personality of each hadron. For instance, the width of $\omega$-meson could be larger as $T$ increases instead of smaller.[47]

Up to now, we have considered the system with zero baryon number, where the chiral restoration and its signals can be seen only near $T_{c}$ just because of the slow decrease of $\langle\bar{q} q\rangle_{T}$. This situation does change once one introduces the finite baryon number, which is the central topic in the next section.

\section{$4 \quad$ QCD at finite density $(T=0, \rho \neq 0)$}

\subsection{Condensates at finite $\rho$}

Let us consider the finite density medium with $T=0$. One of the main differences of this system from the $(T \neq 0, \rho=0)$ system is the behavior of various condensates. For instance, the light quark condensate in the chiral limit decreases slowly at low temperature[48]

$$
\langle\bar{q} q\rangle_{T} /\langle\bar{q} q\rangle_{0}=1-\left[\left(N_{f}^{2}-1\right) / N_{f}\right] T^{2} / 12 f_{\pi}^{2}+\cdots,
$$

while it decreases linearly at low density[49]

$$
\langle\bar{q} q\rangle_{\rho} /\langle\bar{q} q\rangle_{0}=1-\rho \cdot \Sigma_{\pi N} / f_{\pi}^{2} m_{\pi}^{2}+\cdots,
$$

with $\Sigma_{\pi N}=(45 \pm 12) \mathrm{MeV}$ being the $\pi \cdot \mathrm{N}$ sigma term.[50] Note that eqs.(10) and (11) are model independent relations. If one extrapolates the latter formula to normal nuclear matter density $\rho_{0}=0.17 \mathrm{fm}^{-3}$, the condensate decreases almost $20-30 \%$. Accordingly, one can expect rapid change of hadron properties at relatively low densities. 
(Such changes can be seen only near $T_{c}$ in the $(T \neq 0, \rho=0)$ system.; Actually, there are implicit evidences of the partial restoration of chiral symmetry in finite nuclei.[51] 4.2. Hadrons at finite $\rho$

QCD sum rules are the best way to analyse the hadrons in medium (as far as the density is not too high), since it gives a direct relation between the condensates in medium with resonance parameters in a model independent way.

The recent analysis by $\mathrm{Su} \mathrm{H}$. Lee and myself[53] shows that the vector mesons $(\rho$, $\omega$ and $\phi$ mesons) change their properties rapidly at finite density. [54] In this channel, the same current correlation function $T J_{\mu}(x) J_{\nu}(0)$ appears both in QCD sum rules and in the deep inelastic lepton-hadron scattering (DIS). In the low density medium, this operator not only picks up the vacuum part $\left\langle T J_{\mu}(x) J_{\nu}(0)\right\rangle_{0}$ but also picks up the medium part $\left\langle T J_{\mu}(x) J_{\nu}(0)\right\rangle_{N} \cdot \rho$. The latter is related to the parton distribution function of the nucleon in DIS. One can show that the most important medium condensates in the vector channel are

$$
\left\langle\bar{q} \gamma_{\mu} D_{\nu} q\right\rangle_{\rho} \text { and }\langle\bar{q} q\rangle_{\rho}^{2}
$$

for $\rho-\omega$ meson and $m_{s}\langle\bar{s} s\rangle_{\rho}$ for $\phi$-meson.

After carrying out the Borel improvement for the operator product expansion, one finally obtains

$$
M_{V}^{*} / M_{V}=1-C_{V} \cdot\left(\rho / \rho_{0}\right),
$$

with $C_{V} \simeq 0.18(0.15 y)$ for $\rho-\omega$ meson ( $\phi$ meson) and $y$ being the strangeness content of the nucleon $\left(y=\langle\bar{s} s\rangle_{N} /\langle\bar{u} u+\bar{d} d\rangle_{N}=0.12-0.22\right)[52]$.

\subsection{Implications of in-medium modification of hadrons}

Many implicit evidences of the dropping rho-omega mass in finite nuclei are extensively discussed in Ref.[51] which include the $K^{+}$scattering off medium-heavy nuclei, polarization observables in nucleon-nucleus scattering, enhancement of the spin-orbit part of the G-matrix element, and enhancement of tensor-force due to $\rho$-meson in medium. These phenomenological studies require $C_{V} \sim 0.15$ which is comparable to our value in eq.(13). In this sense, our result of the QCD sum rule supports such phenomenological idea.

In the relativistic heavy ion collisions, one can even expect to detect the modification of hadrons by measuring the leptonic decay of rho, omega and $\phi$ mesons, [55] the $2 \pi$ correlation from the decay of the scalar meson $[21,44]$ and the enhancement of the kaon production.[56] In particular, only a few \% change of the $\phi$-meson mass can affect its decay property drastically, since $m_{\phi}$ is very close to the threshold of its main decay mode $\left(K^{0} K^{0}\right.$ and $\left.K^{+} K^{-}\right)$. [55] Eq.(4.4) suggests that $\phi$-meson is going to be more stable in medium, if the kaon mass $\left(m_{K}\right)$ does not change in medium as some of the effective models claim[22]. As a result, the leptonic yield

$$
R=N_{\phi} /\left(N_{\rho}+N_{\omega}\right),
$$

will be enhanced in high density/temperature medium. 
As we have already discussed, one can see the considerable change of $M_{V}^{*}(T)$ only near the critical point, while $M_{V}^{*}(\rho)$ has $O(20 \%)$ change eicn at $\rho_{0}$. Therefore, the finite density system ( ion collisions) will be a better place to see the effect of the partial restoration of chiral symmetry and changes of hadron properties in experiment.

\subsection{Phase transition to quark matter}

There have been proposed numbers of scenarios and various exotic phases as intermediate states between nuclear (or neutron) matter and quark matter. They include charged and neutral pion condensation, [57] the kaon condensation, [58] the parity doubling matter, [59] massive quark matter[60] and so on. Unfortunately, none of these phases is not confirmed yet since the place to test the idea has been so far confined in neutron sta s (the mass-radius relation, pulsation frequencies and glitches, cooling of the surface temperature and so on).[61] Therefore, even a little information from the heavy ion collisions in laboratory is welcome.

Instead of going into the details of the above scenarios, let us get back to a primitive idea of the phase transition from nuclear matter to quark matter. The nucleon has a radius of order $R=\sqrt{\left.{ }^{-2}\right\rangle} \simeq 0.8 \mathrm{fm}$ and the inter nucleon distance in nuclear matter at $\rho_{0}=0.17 \mathrm{fm}^{-3}$ is $\sim 2.5 \mathrm{fm}$. A simple geometrical argument tells us that nucleons are packed closely at

$$
\rho_{\mathrm{cl}}=\left(4 \pi R^{3} / 3\right)^{-3} \sim 3 \rho_{0},
$$

and the complete overlapping occurs at $\rho_{\mathrm{c} 2} \sim 6 \rho_{0}$ where the mean nucleon distance $l_{d} \simeq 1.4 \rho^{-1 / 3}$ becomes comparable to $R$. In higher densities than $\rho_{c 1}$, one cannot neglect the internal structure of the nucleon, since the nucleons start to overlap $\left(l_{d}<\right.$ $2 R$ ). In other words, there is no a priori reason to believe in models based on the point-like structure of nucleon above this density. As the recent development of the non-perturbative QCD tells us, nucleon can be described either as a 3-quark bound state with $R \sim 0.8 \mathrm{fm}$ or as a chiral soliton having the same size. The seeming difference between the two pictures merely stems from the different choice of variables to describe the same physics. At extremely high $\rho$, the quark-gluon language is certainly a most effective way to describe the system. Then, as one decreases $\rho$ toward $\rho_{c 1}$, the non-perturbative physics starts to emerge, which is similar to the situation we have encountered in section 2 . In the quark-gluon picture, the matter with density around $\rho_{c 1} \sim \rho_{c 2}$ will be a strongly correlated quark matter, while it is a system of pions with non trivial topology in the hadron picture.[62]

Now there is another kind of interesting possibility without going into such strong coupling system above $\rho_{c 1}$, which I will call multi-clustering scenario. [63] Suppose that one of the 6-quark channels can form compaci ( $\sim$ size of nucleon) and not two high mass $\left(\sim 2 M_{N}\right)$ state in medium. (It does not have to be stable in the free space). Then, instead of increasing the baryon density, one can form a low density bose gas composed of the 6-quark state. Namely, it occurs the iocalization of the 
quark density instead of distributing it to entire space. One of the candidate of such 6 -quark state is the $\mathrm{H}$-dihyperon whose mass is possibly not too higher than $2 M_{N}$ even in the free space. One can also generalize the idea to a hierarchy of the similar phase transitions: nuclear matter $\rightarrow 6$-quark matter $\rightarrow 12$-quark matter $\rightarrow 18$-quark matter $\rightarrow \cdots$, which is an interesting alternative path toward quark matter. A similar scenario has been also proposed by Kagiyama et al.[64].

\section{Concluding remarks}

We have looked at the physics in the $(T, \rho)$ plane on the basis of various approaches; perturbative QCD, lattice QCD, effective theories and QCD sum rules. None of them is encugh powerful to cover all the region of the phase diagram. However, recent studies are making their applicable range wider and we have already learned some interesting physics related to the non-perturbative nature of QCD.

(1) Non-perturbative physics above $T_{c}$ :

The higher order analyses of the perturbation theory have shown that there exists non-perturbative physics even above $T_{c}$. The lattice data of the free energy and also the screening mass in the scalar-pseudoscalar channel seem to support the coexistence of perturbative and non-perturbative physics above $T_{c}$. There are several ideas about the physics behind them, which include the non-perturbative cutoff, the gluon condensate above $T_{c}$, color singlet soft modes above $T_{c}$ and so on. Clear physical understanding of them are called for.

(2) Chiral restoration and its associated phenomena:

$\langle\bar{q} q\rangle$ for light quarks is a good order parameter near the chiral limit. There is little doubt about the existence of chiral transition at high $T$ in the chiral limit and probably it is also true at high $\rho$. Like similar cases in condensed matter and nuclear physics, we can expect phenomena associated with this phase transition; in particular the modification of the elementary modes of excitations (hadrons) in medium. QCD sum rules and effective theories are suitable to calculate such modifications at least at relatively low $T$ and $\rho$. It turns out that $\langle\bar{q} q\rangle$ and hence the hadron properties change much faster at finite $\rho$ than at finite $T$. This suggests a right place to see the effects in the relativistic heavy ion collisions: To see the quark-gluon plasma clearly, one should look at the high $T$ region, while to see the chiral restoration and its associated phenomena, one should look at the high density region. Lepton pairs from the vector mesons and the $2 \pi$ correlation from the scalar meson are the useful probes in the latter case.

(3) Transition from nuclear matter to quark matter:

Nothing definite is known for this transition although many possible intermediate 
phases are proposed such as the pion and kaon condensation, the parity doubling matter, skyrmion crystal and so on. One of the interesting recent ideas is the multiclustering scenario where the bose condensation of multi-quark states (such as $H$ dihyperon) is formed to avoid the high nucleon density. 


\section{References}

[1] J. C. Collins and M. J. Perry, Phys. Rev. Lett. 34 (1975) 1353.

A. B. Kisslinger and P. D. Morley, Phys. Rev. D13 (1976) 2771.

[2] A. D. Linde, Phys. Lett. 96B (1980) 289. E. Shuryak, Phys. Rep. 61 (1980) 72.

D. J. Gross, R. D. Pisarski and L. G. Yaffe, Rev. of Mod. Phys. 53 (1981) 43.

C. DeTar, Phys. Rev. D32 (1985) 276.

[3] J. Kapsta, Nucl. Phys. B148 (1979) 461.

[4] R. D. Pisarski and E. Braaten, Nucl. Phys. B337 (1990) 569, ibid. B339 (1990) 199.

[5] S. Nadkarni, Phys. Rev. D33 (1986) 3738; ibid. D34 (1986) 3904, and references therein.

[6] J. Engels, J. Fingberg, F. Karsch, D. Miller and M. Weber, Phys. Lett. 252B (1990) 625.

[7] For the previous simulations, see the review, A. Ukawa, Nucl. Phys. A498 (1989) $227 \mathrm{c}$.

[8] Y. Deng, Nucl. Phys. B (Proc. Suppl.) 9 (1989) 334.

[9] J. Engels, J. Fingberg, K. Redlich, H. Satz and M. Weber, Z. Phys. C42 (1989) 341.

[10] M. I. Gorenstein and O. A. Mogilevsky, Phys. Lett. 228B (1989) 121.

M. I. Gorenstein, O. A. Mogilevsky and S. Mrowczynski, Phys. Lett. 246B (1990) 200.

[11] M. Campostrini and A. Di Giacomo, Phys. Lett. 197B (1987) 403.

[12] Su H. Lee, Phys. Rev. D40 (1989) 2484.

R. J. Furnstahl, T. Hatsuda and Su H. Lee, Phys. Rev. D42 (1990) 1744.

[13] C. Adami, T. Hatsuda and I. Zahed, Phys. Rev. D43 (1991) 921.

[14] Su H. Lee and T. Hatsuda, under investigation.

[15] C. DeTar and J. Kogut, Phys. Rev. D36 (1987) 2828.

[16] See e.g., Y. Koike, M. Fukugita and A. Ukawa, Phys. Lett. B213 (1988) 497.

S. Gottlieb et al., Phys. Rev. Lett. 59 (1987) 1881.

[17] V. L. Eletskii and B. L. Ioffe, Sov. J. Nucl. Phys. 48 (1988) 384.

[18] G. E. Brown, private communication.

E. Shuryak, private communication.

[19] Y. Koike and T. Hatsuda, under investigation.

[20] S. Gottlieb et al., Phys. Rev. Lett. 59 (1987) 1881. 
[21] T. Hatsuda and T. Kunihiro, Phys. Rev. Lett. 55 (1985) 158; Prog. Theor. Phys. 74 (1985) 765; Prog. Theor. Phys. Suppl. 91 (1987) 284; Phys. Lett. 185B (1987) 304.

[22] T. Kunihiro, Nucl. Phys. B351 (1991) 593.

[23] T. Kunihiro, preprint RYU-THP-91/4 (1991), Phys. Lett. B in press.

[24] A. Ukawa, Nucl. Phys. B (Proc. Suppl.) 10A (1989) 66.

[25] M. Fukugita, M. Okawa and A. Ukawa, Phys. Rev. Lett. 63 (1989) 1768, Nucl. Phys. B337 (1990) 181.

[26] F. R. Brown et al., Phys. Rev. Lett. 65 (1990) 2491.

[27] R. D. Pisarski and F. Wilczek, Phys. Rev. D29 (1984) 338.

S. Midorikawa, H. So and S. Yoshimoto, Z. Phys. C34 (1987) 307.

[28] E. M. Ilgerifritz and E. V. Shuryak, Nucl. Phys. B319 (1989) 511.

[29] T. Hatsuda and T. Kunihiro, Phys. Lett. 198B (1987) 126.

[30] T. Hatsuda and T. Kunihiro, Phys. Rep. (to be published).

[31] See e.g. M. Fukugita, S. Ohta, Y. Oyanagi and A. Ukawa, Phys. Rev. Lett. 58 (1987) 2515.

[32] P. Gerber and H. Leutwyler, Nucl. Phys. B321 (1989) 387.

[33] J. Cleymans, A. Kocić and M. D. Scadron, Phys. Rev. D39 (1989) 323.

A. Kocić, Phys. Rev. D33 (1986) 1785.

[34] R. D. Pisarski, Phys. Lett. 110B (1982) 155.

[35] A. L. Bochkarev and M. E. Shaposhnikov, Nucl. Phys. B268 (1986) 220.

[36] T. Hatshimoto, O. Miyamura, K. Hirose and T. Kanki, Phys. Rev. Lett. 57 (1986) 2123.

[37] T. Matsui and H. Satz, Phys. Lett. B178 (1986) 416.

[38] T. Hatsuda, Nucl. Phys. B (Proc. Suppl.) 23B (1991) pp.368-371.

[39] See, S. Narison, QCD Spectral Sum Rules (World Scientific, Singapore, 1989).

[40] H. G. Dosch and S. Narison, Phys. Lett. 203B (1988) 155.

R. J. Furnstahl, T. Hatsuda and Su H. Lee, Phys. Rev. D42 (1990) 1744.

[41] C. Gale and J. I. Kapsta Nucl. Phys. B357 (1991) 65.

H. Leutwyler and A. V. Smilga, Nucl. Phys. B342 (1990) 302.

[42] Y. Takahashi and H. Umezawa, Collective Phenomena, 2 (1975) 55.

[43] In terms of the chiral Lagrangian, this corresponds to the $T$ dependence of the kinetic term $(1 / 2)\left(f_{\pi}^{*} \partial \pi\right)^{2}$.

[44] Y. Takahashi and S. Nagamiya, Nucl. Phys. A52s (1991) 623c. 
[45] R. W. Minich and L. R. Ram Mohan, Phys. Rev. D19 (1979) 1582.

T. Hashimoto, K. Hirose, T. Kanki and O. Miyamura, Phys. Rev. D37 (1988) 3331 .

[46] T. Hashimoto, K. Hirose, T. Kanki and O. Miyamura, Nucl. Phys. A478 (1988) 687.

H. Kikuchi and T. Akiba, Phys. Lett. 200B (1988) 543.

[47] E. V. Shuryak, private communication.

[48] J. Gasser and H. Leutwyler, Phys. Lett. 188B (1987) 477.

[49] E. G. Drukarev and E. M. Levin, Preprint LPTHE Orsay 90/52, (1990).

[50] J. Gasser, H. Leutwyler and M. E. Sainio, Phys. Lett. 253B (1991) 252.

[51] G. E. Brown, in Proceedings of From Fundamental Fields to Nuclear Phenomena, (Univ. of Colorado, Boulder, Sept. 20-22, 1990); Nucl. Phys. A522 (1991) 397c.

[52] T. Hatsuda and T. Kunihiro, Preprint CERN-TH.5836/90 (1990) Nucl. Phys.B (1991) in press.

[53] T. Hatsuda and Su H. Lee, preprint YSTP-91-10, 40561-05-INT-00-02, July (1991).

T. Hatsuda, Su H. Lee and Y. Koike, in preparation.

[54] As for the nucleon sum rule, a consistent treatment has been recently developed in T. Hatsuda, H. Høgaasen and M. Prakash, Phys. Rev. Lett. 66 (1991) 2851. One of the main conclusion of this work is that there are two kinds of self energies (scalar and vector) which cancel out with each other to make the pole position of the nucleon propagator almost insensitive to density (each contributions are large though.) This has been suggested for a long time in effective theories such as the $\sigma-\omega$ model and the Dirac phenomenology for a long time without solid QCD basis.

[55] R. D. Pisarski, Phys. Lett. B110 (1982) 155; in Proceedings of Quark Matter '90, Nucl. Phys. A525 (1991).

E. V. Shuryak, Nucl. Phys. A525 (1991) 3c.

[56] C. M. Ko, Z. G. Wu, L. H. Xia and G. E. Brown, Phys. Rev. Lett. 66 (1991) 2577.

[57] See, T. Muto and T. Tatsumi, Prog. Theor. Phys. 83 (1990) 499 and references therein.

[58] D. B. Kaplan and A. E. Nelson, Phys. Lett. 175B (1986) 57; G. E. Brown, K. Kubodera and M. Rho, Phys. Lett. 192B (1987) 273; T. Taisumi, Prog. Theor. Phys. 80 (1988) 22.

[59] C. DeTar and T. Kunihiro, Phys. Rev. D39 (1989) 2805.

T. Hatsuda and M. Prakash, Phys. Lett. 224B (1989) 11. 
[60] T. Hatsuda, Prog. Theor. Phys. 75 (1986) 301.

[61] R. Tamagaki, Kyoto preprint KUNS-1063 (1991), in Proceedings of The Structure and Evolution of Neutron Stars (SENS '90), (Kyoto, Japan, Nov. 6-10, 1990). T. Takatsuka, in Condensted Miatter Theories, Vol.3, ed. by J. S. Arponen et al., (Plenum Publishing, 1988).

[62] L. Castillejo, P. S. J. Jones and A. D. Jackson, Nucl. Phys. A501 (1989) 801.

[63] R. Tamagaki, Prog. Theor. Phys. 85 (1991) 321.

[64] S. Kagiyama, S. Hirooka, H. Kikukawa and J. Kikukawa, Prog. Theor. Phys. 69 (1983) 579. 
I would like to thank Prof. D. P. Min for giving me an oppotunity to give lectures at this winter school and for his worm hospitality. I also thank S. Aoki, G. Brown, T. Kunihiro, Y. Koike, S.-H. Lee, M. Rho, K. Yazaki and many other participants of the school for fruitful discussions. This work was supported by U.S. Department of Energy under grant No. DE-FG06-90ER40561. 

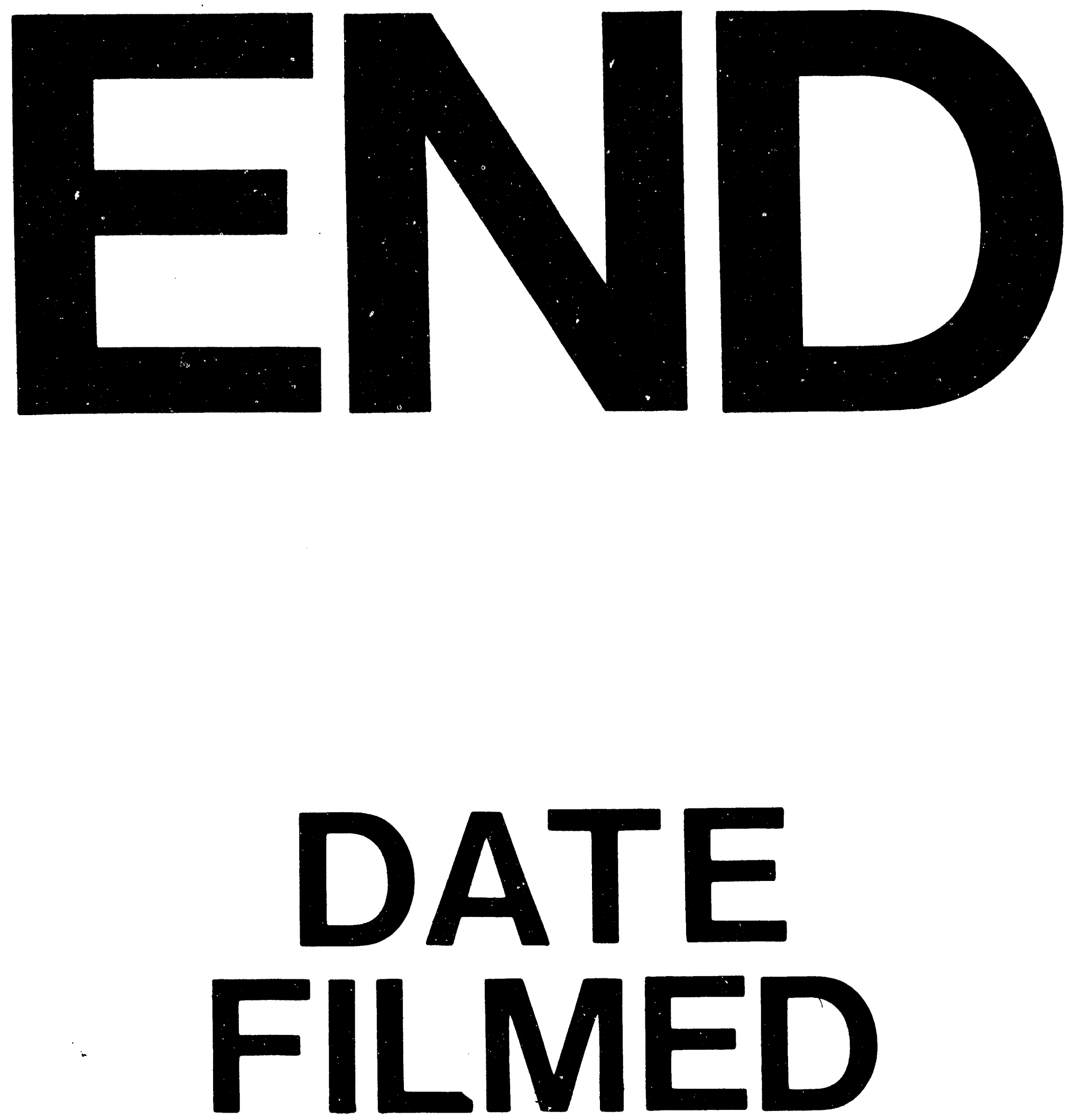

1

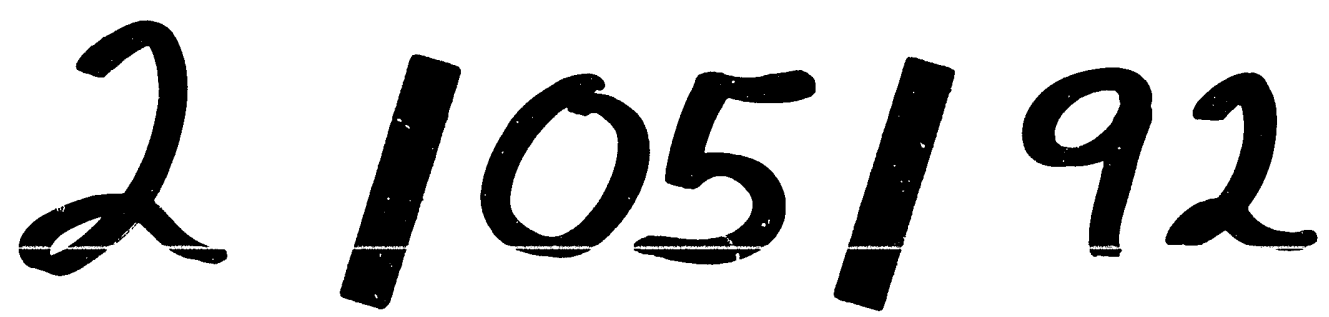


